\title{
Finite strain elastoplasticity considering the Eshelby stress for materials undergoing plastic volume change
}

\author{
Bennett, K.C. ${ }^{a}$, Regueiro, R.A. ${ }^{\mathrm{b}, *}$, Borja, R.I. ${ }^{\mathrm{a}}$ \\ a Stanford University, Stanford, CA, 94305, USA. \\ ${ }^{b}$ University of Colorado Boulder, Boulder, CO, 80309, USA. E-mail: richard.regueiro@Colorado.edu
}

\section{Abstract}

In consideration of materials capable of undergoing significant plastic changes in volume, an alternative finite strain hyper-elastoplastic constitutive framework is proposed in terms of the Eshelby stress. Taking a phenomenological point of view, a thermodynamically-consistent approach to developing the constitutive equations is presented and discussed. Various Eshelby-like stresses are defined and shown to be energyconjugate to the plastic velocity gradient. A general framework is formulated in the stress-free/plasticallydeformed intermediate configuration associated with the multiplicative split of the deformation gradient, as well as the current configuration. A novel Eshelby-like stress measure is proposed, which is scaled by the elastic Jacobian, and is shown to be energy-conjugate to the plastic velocity gradient in the spatial representation. Modified Cam-Clay and Drucker-Prager cap plasticity constitutive equations are introduced, and large strain isotropic compression simulations are performed and compared with experimental measurements. The model results are compared with standard approaches formulated in terms of the Mandel and Kirchhoff stresses, which are shown to require the assumption of isochoric plasticity to satisfy the Clausius Planck inequality (Mandel) and preserve that the intermediate configuration remains stress-free (Kirchhoff). The simulations show that both the material and spatial Eshelby-like stress measures presented here produce the same mean Cauchy stress results; whereas, standard formulations which make use of isochoric plasticity assumptions, diverge from each other at significant plastic volume strains. Standard formulations are further shown to violate the second law of thermodynamics under certain loading conditions. Calibration of model parameters to high pressure isotropic compression of Boulder clay is used to compare the various models.

Keywords: A. thermomechanical processes, B. finite strain, B. geological material, B. constitutive 8 behavior, bulk plasticity

\section{Introduction}

Eshelby's notion of what he termed the "energy momentum tensor" (Eshelby, 1951, 1956, 1975), which has since come to be known as the "Eshelby stress," has found applications in numerous aspects of finite strain elastoplasticity. Epstein and Maugin (1990) identified the Eshelby stress tensor with what they termed "torsion of the material connection" due to configurational inhomogeneity which arises from the elastic relaxation of a crystal structure containing dislocations. This concept was later cast as a more formal statement of elastoplasticity, that it is the Eshelby stress which is the driving force of inelastic deformation (Maugin, 1994; Maugin and Epstein, 1998; Cleja-Tigoiu and Maugin, 2000; Maugin, 2013), showing that under isochoric plastic deformation for metals (with which they were concerned) the so called static Eshelby stress coincides up to a sign with the Mandel stress (Mandel, 1974). Gurtin (1995) showed through the second law of thermodynamics that the Eshelby stress is the pertinent stress measure for a continuum body subjected to what he coined "configurational forces," making the important observation that bulk

* Corresponding author 
tension can be equated with the bulk free energy. The concept of configurational (or "material") forces and the notion of the Eshelby stress have proven useful in describing the concept, generally attributed to Lee (1969), of a stress-free/plastically-deformed intermediate configuration, especially in view of local strain compatibility (Menzel and Steinmann, 2007; Svendsen et al., 2009), and has been applied to (finite) damage mechanics(e.g., Brünig, 2004; Monchiet et al., 2014), as well as fracture mechanics, (e.g., Maugin, 1994; Kalpakides and Dascalu, 2002; Miehe and Gürses, 2007; Stumpf et al., 2009, among others). The Eshelby stress has also been found to have applications in the modeling of finite volumetric growth (e.g., Epstein and Maugin, 2000; Garikipati et al., 2004; Ganghoffer, 2010, 2013), and micromorphic plasticity, (e.g., Lee et al., 2014)).

Despite the fact the Eshelby stress has found much success in finite strain inelastic applications, especially with respect to inhomogeneity, damage and fracture, relatively little attention has otherwise been given to the notion of the Eshelby stress in the development of a hyper-elastoplastic constitutive framework for those materials that may experience large volumetric plastic deformations, such as geomaterials. This may be in part because, as we show in detail here, the Eshelby stress (when compared to the Kirchhoff stress in the current configuration (with respect to reference volume) or the Mandel stress in the intermediate configuration, for example) becomes fundamentally important to a thermodynamically-consistent derivation of the constitutive equations for that class of materials which is capable of undergoing significant plastic volume change. For example, Simo and Ortiz (1985); Simo $(1985,1998)$ identified a second Piola-Kirchhoff stress $\overline{\boldsymbol{S}}$ - conjugate to a "metric" in the form of the right elastic Cauchy-Green tensor $\overline{\boldsymbol{C}}^{e}$ applied to the plastic velocity gradient $\overline{\boldsymbol{L}}^{p}$ —as the pertinent stress measure within the intermediate configuration under the assumption of isochoric plastic flow. Sansour et al. (2006, 2007), similarly for the isochoric case, identified what they recognized as an Eshelby-like tensor (coinciding up to a spherical term) for formulation of the flow rule for anisotropic materials - for which the intermediate configuration is identified as the proper one in which to formulate finite strain elastoplastic anisotropy.

Geomaterials are known to exhibit significant plastic volume change; however, many of the advancements with respect to finite strain elastoplasticity of geomaterials have focused on a Kirchhoff or Cauchy stress form (e.g., Nemat-Nasser, 1983; Hashiguchi, 1993; Simo and Meschke, 1993; Borja and Tamagnini, 1998; Callari et al., 1998; Jeremić et al., 2001; Borja, 2013), which is generally valid only for material isotropy (i.e., no texture effects) and for the assumption of small plastic volume strain. Recently, Balieu and Kringos (2015) formulated finite strain elastoplasticity with anisotropic damage, assuming a multiplicative decomposition of the deformation gradient. Attention was paid to the thermodynamics and mappings of quantities between the various configurations (reference, intermediate, effective-damaged intermediate, and current configurations), assuming that the Helmholtz free energy per unit volume of the plastically-deformed intermediate configuration, and plastically-deformed effective intermediate configuration with damage, are equivalent. By mapping from one configuration to the next, it can be shown that the total energy integrated over the respective volumes is equivalent (starting in the current configuration), but if there is inelastic volumetric deformation between the configurations (as there would be expected for geomaterials with volumetric plasticity and/or volumetric damage), then the per unit volume free energies of the intermediate and reference configurations will not be the same, giving rise to Eshelby-like stresses being energy conjugate to the plastic velocity gradient represented in either the intermediate or current configurations, as will be discussed in the paper. This also relates to the choice of constitutive parameters in the respective configurations, which is also discussed herein. For small strain experimental data (e.g., that considered by Balieu and Kringos (2015) of $<2 \%$ ), the choice of stress measure will not be critical. We show for our model formulations with Eshelby-Mandel stress (and other stresses) that at large volumetric deformation of Boulder clay (up to $27 \%$ ), the choice of stress measure in the constitutive model is important.

Many papers in the literature have considered finite strain elastoplastic constitutive models for materials that may exhibit significant plastic volumetric deformation, yet almost all neglect consideration of the Eshelby stress and are formulated in the current configuration in terms of the Kirchhoff stress (and therefore limited to the assumption of material isotropy and implicitly (as will be shown) making the assumption that the plastic volumetric deformation is small). Other papers in the literature have considered plasticity models at finite strain formulated in the intermediate configuration for materials that may exhibit anisotropic constitutive response (i.e., inherent texture, and/or deformation-induced anisotropy), yet almost all are 
limited to the assumption of isochoric plastic deformation. For example, Ortiz and Pandolfi (2004) presented a Cam-Clay theory at finite strain, taking a variational approach (plastic flow rule defined, with yield surface derived), assuming a multiplicative decomposition of the deformation gradient. The theory was represented in the current configuration, and so was limited to isotropic materials: there was no discussion of the per-unitvolume, plastically-deformed, intermediate configuration. Mosler and Bruns (2009) extended the variational framework for non-associative plasticity and volumetric-deviatoric splits of elastic and plastic deformations. They identified the Mandel stress as the thermodynamically-conjugate stress. For saturated porous media at finite strain, Gajo (2011) presented a finite strain elastoplastic constitutive model with compressible solid skeleton and compressible solid and fluid constituents. Constitutive equations were postulated in the intermediate configuration, identifying the Second Piola-Kirchhoff stress in the intermediate configuration as thermodynamically-conjugate to an inelastic deformation measure. For the case of volume preserving plasticity only, Shutov and Ihlemann (2014) recently provided a comparison of various approaches to finitestrain (isochoric) plasticity, and much attention continues to be given to anisotropic elastoplastic deformation at finite strain for those materials that do not undergo plastic volume change (e.g., Vladimirov et al., 2010; Brepols et al., 2014; Clausmeyer et al., 2014). Miehe (1998) presented general Lagrangian and Eulerian forms of the constitutive equations for anisotropic elastoplastic materials at finite strain, accounting for material frame invariance. He formulated "plastic forces" that are energy-conjugate to appropriate deformation measures, one such plastic force being the Mandel stress. It would appear that such general treatment by Miehe (1998) for anisotropic materials at finite strain could have identified the Eshelby stress as a plastic force for materials with volumetric plastic deformation, but such identification was not made explicitly. Borja and Tamagnini (1998) presented an extension of a small-strain hyperelastic Cam-Clay plasticity model to finite strain, taking a multiplicative decomposition of the deformation gradient and representing the constitutive equations in terms of the Kirchhoff stress, and stored energy with respect to the reference configuration.

In the following, we present a finite strain elastoplastic canonical constitutive framework for materials capable of undergoing significant volumetric plastic deformation formulated in terms of the Eshelby stress in the intermediate configuration, allowing for the possibility of elastic and plastic anisotropy, and also the current configuration (restricted to isotropy). We develop this framework with specific application to the constitutive modeling of geomaterials in mind; however, we note that the results presented here may be applicable to any material capable of undergoing inelastic volumetric strain, such as powders, some biologic materials (e.g,. bone), metallic foams, and filled polymers, to name just a few. We adopt a phenomenological view of the multiplicative split of the deformation gradient, which gives rise to the concept of the plastically-deformed elastically-relaxed intermediate configuration, and show that various Eshelby-like stresses are energy-conjugate to the plastic velocity gradients in their respective configurations according to the second law of thermodynamics. In Section 2, we give a brief overview of finite deformation kinematics and the adopted notation, and then in Section 3 we derive the canonical forms of the constitutive equations in the intermediate and current (spatial) configurations for the case of non-isochoric plasticity (i.e., considering plastic changes in volume and, in turn, mass density), and also provide for comparison the corresponding forms applicable to the assumption of isochoric plasticity (also called isochoric flow). An important motivation of the present paper is to provide (for materials capable of large plastic volume change) the general intermediate configuration formulation of the constitutive equations which allow for the introduction of elastic and plastic anisotropy. Specific classical choices of free energy and yield functions are examined in Section 4, including Drucker-Prager and Modified Cam-Clay plasticity formulated in the intermediate and current configurations, respectively, and their implications with respect to plastic changes in volume are considered. In Section 5 results are compared to recently published measurements of highpressure/large-strain isotropic compression tests. The numerical results clearly show that what has become accepted as the standard implementation of finite strain elastoplasticity can under-predict volumetric strain at a given mean Cauchy stress for the case of materials (such as geomaterials) that plastically change volume, and can violate the second law of thermodynamics under certain loading conditions. We conclude in Section 6 with a discussion of the implications of the concepts presented here, and give a brief description of ongoing research. 


\section{Kinematics}

To start, a brief summary is provided of the kinematics of finite strain elasticity and elastoplasticity, and the notation adopted. For a more detailed description of finite deformation kinematics, the reader is referred to one of the many books on the subject (cf. Truesdell and Toupin (1960); Truesdell and Noll (1965); Eringen (1967); Malvern (1969); Marsden and Hughes (1983); Holzapfel (2000) for nonlinear elasticity, and Hashiguchi and Yamakawa (2013) for elastoplasticity). Tensors are represented in boldface (a.k.a., direct notation), and summation convention is adopted for repeated indices unless specified otherwise. Referring to Fig.1, a mapping $\varphi_{t}: \mathscr{B}_{0} \rightarrow \mathscr{B}_{t} \subset \mathbb{R}^{3}$ maps each material point $P$ in the reference configuration $\mathscr{B}_{0}$ (also called the "Lagrangian" or "material" configuration) to a point $p=\varphi_{t}(P)$ in $\mathscr{B}_{t}$ (also called the "Eulerian" or "spatial" configuration) at time $t$, i.e. $\mathscr{B}_{t}=\varphi_{t}\left(\mathscr{B}_{0}\right)=\varphi\left(\mathscr{B}_{0}, t\right)$. The position of $P \in \mathscr{B}_{0}$ relative to an origin $O$ is described in terms of the Cartesian basis $\boldsymbol{E}_{A}$ by the position vector $[\boldsymbol{X}]_{\left\{\boldsymbol{E}_{A}\right\}}=$ $X_{A} \boldsymbol{E}_{A}=X_{1} \boldsymbol{E}_{1}+X_{2} \boldsymbol{E}_{2}+X_{3} \boldsymbol{E}_{3}$. Likewise, a point $p \in \mathscr{B}_{t}$ is represented by position vector $[\boldsymbol{x}]_{\left\{\boldsymbol{e}_{a}\right\}}=$ $x_{a} \boldsymbol{e}_{a}=x_{1} \boldsymbol{e}_{1}+x_{2} \boldsymbol{e}_{2}+x_{3} \boldsymbol{e}_{3}$. The Cartesian bases corresponding to each configuration will be implied, and, for example, typically represent points in $\mathscr{B}_{0}$ by $\boldsymbol{X}$ or in indicial (component) notation as $X_{A}$. For a more general treatment of the kinematics of elastoplasticity in general curvilinear coordinates, refer to Le and Stumpf (1993); Steinmann (1996); Clayton et al. (2004); Clayton (2011), among others.

The position of a point at $\mathscr{B}_{t}$ is hence described by $\boldsymbol{x}=\boldsymbol{\varphi}(\boldsymbol{X}, t)$ or equivalently in indicial notation as $x_{a}=\varphi_{a}\left(X_{1}, X_{2}, X_{3}, t\right)$; at places in the paper both notations (boldface and indicial) will be given, but typically only the one will be given which is judged to be the clearest for the case at hand. The deformation can further be specified by the displacement vector field $\boldsymbol{u}(\boldsymbol{X}, t)$, as

$$
\boldsymbol{x}=\boldsymbol{\varphi}(\boldsymbol{X}, t)=\boldsymbol{X}+\boldsymbol{u}(\boldsymbol{X}, t) .
$$

The deformation gradient is written as

$$
\boldsymbol{F}=\boldsymbol{F}(\boldsymbol{X}, t):=\frac{\partial \varphi(\boldsymbol{X}, t)}{\partial \boldsymbol{X}}, \quad F_{a A}=F_{a A}\left(X_{1}, X_{2}, X_{3}, t\right):=\frac{\partial \varphi_{a}\left(X_{1}, X_{2}, X_{3}, t\right)}{\partial X_{A}},
$$

such that an infinitesimal line segment $d \boldsymbol{X}$ is mapped from $\mathscr{B}_{0}$ to $\mathscr{B}_{t}$ by

$$
d \boldsymbol{x}=\boldsymbol{\varphi}_{t}(d \boldsymbol{X})=\boldsymbol{F} d \boldsymbol{X} .
$$

The concept of the multiplicative split of the deformation gradient $\boldsymbol{F}=\boldsymbol{F}^{e} \boldsymbol{F}^{p}$ is assumed (see Figure 1), associated with the concept of the plastically-deformed and elastically-relaxed intermediate configuration $\overline{\mathscr{B}}$ (Lee and Liu, 1967; Lee, 1969). The multiplicative decomposition has utility for the following reasons: (1) it retains hyper-elastic constitutive relations (isotropic and anisotropic) through $\boldsymbol{F}^{e}$ (Ogden, 1984); (2) it has physical basis for crystal elastoplasticity (Asaro, 1983; Clayton, 2011; Clayton et al., 2014); (3) it is the proper configuration in which to formulate phenomenological anisotropic elastoplasticity at finite strain given its elastically-unloaded intermediate configuration (cf. Svendsen, 2001; Svendsen et al., 2006; Sansour et al., 2007); among others); (4) it provides a natural framework for studying dislocation defect mechanics of crystalline materials (Kondo, 1952; Bilby et al., 1955; Steinmann, 1996; Bammann, 2001; Regueiro et al., 2002; Clayton et al., 2006; Clayton, 2011); and (5) numerically it provides a naturally objective time integration scheme (Simo and Ortiz, 1985). Upper-case letters are used to denote quantities and indices in $\mathscr{B}_{0}$ (e.g., differential line segment $d \boldsymbol{X}=d X_{A} \boldsymbol{E}_{A}$ ), upper-case with an over-bar (e.g., $d \overline{\boldsymbol{X}}=$ $\left.d \bar{X}_{\bar{A}} \overline{\boldsymbol{E}}_{\bar{A}}\right)$ for those in $\overline{\mathscr{B}}$, and lower-case letters for those in $\mathscr{B}_{t}$ (e.g., $d \boldsymbol{x}=d x_{a} \boldsymbol{e}_{a}$ ). Tensors can hence be represented as boldface or in component form and their bases are implied.

The Jacobian is defined as the determinant of the deformation gradient $J=\operatorname{det}[\boldsymbol{F}]=J^{e} J^{p}$, and we make note of the Jacobian maps of differential volumes

$$
\begin{aligned}
& d v=J d V=J^{e} d \bar{V} \\
& d \bar{V}=J^{p} d V=J^{e^{-1}} d v .
\end{aligned}
$$


If it is assumed balance of mass $m$ in the current configuration is satisfied for a region comprised of a single phase material, it follows that the Jacobian maps the mass density $\rho=d m / d v$ as follows:

$$
\begin{array}{r}
\rho_{0}=J \rho=J^{p} \bar{\rho}, \\
\bar{\rho}=J^{e} \rho=J^{p^{-1}} \rho_{0},
\end{array}
$$

where the Lagrangian density $\rho_{0}=d m / d V$ is the differential mass per differential reference volume; conservation of mass leads to $D \rho_{0} / D t=0$.

The material time derivative $D(\bullet) / D t$ is also expressed in shorthand notation as $\dot{\overline{(\bullet)}}$. Shorthand notation for the partial derivative is also utilized; for example, the partial derivative with respect to $\boldsymbol{x}$ can be expressed as either $\partial(\bullet) / \partial \boldsymbol{x}$ or $\partial_{\boldsymbol{x}}(\bullet)$. The spatial gradient, further, is also expressed by the symbol $\boldsymbol{\nabla}_{x}$. We have already mentioned the matrix determinant operation $\operatorname{det}[(\bullet)]$, and we similarly denote the trace of a matrix as $\operatorname{tr}[(\bullet)]$. The mean stress is defined for any stress measure $(\bullet)$ as $p^{(\cdot)}=1 / 3 \operatorname{tr}[(\bullet)]$, and the deviatoric stress as $\operatorname{dev}[(\bullet)]=(\bullet)-p^{(\cdot)} \mathbf{1}$.

\section{Thermodynamic formulation of the constitutive equations}

In this section, the second law of thermodynamics in the form of the Clausius-Duhem inequality is presented in order to provide a thermodynamically-consistent formulation of the constitutive equations. First, the general form is developed in the intermediate configuration, allowing for material anisotropy and both isotropic and kinematic hardening. Then the assumption of material isotropy is made in order that the current configuration (spatial) formulation of the constitutive equations may be provided directly (i.e., not mapped from the intermediate configuration). Also provided, for the sake of comparison, is what has become accepted as a "standard" method for hyper-elastic and isochoric plasticity formulations, where the Kirchhoff stress is employed to enforce the second law with respect to the reference volume. This Kirchhoff stress formulation requires that the free energy moduli be defined as constant with respect to unit volume of $\mathscr{B}_{0}$ (not $\overline{\mathscr{B}}$ ), which is problematic when plastic volume change is considered, as will be discussed in detail in Section 4. Finally, we provide a thermodynamically-consistent spatial representation of the constitutive equations with respect to the intermediate configuration unit volume, identifying the pertinent spatial Eshelby-like stress measure for large deformation compressible plasticity.

\subsection{Representation in the intermediate configuration}

The second law of thermodynamics may be expressed as the Clausius-Duhem (C-D) inequality for the rate of internal entropy production at any time $t$ in the current configuration of a continuum body $\mathscr{B}_{t}$ (cf. Truesdell and Toupin, 1960; Holzapfel, 2000),

$$
\int_{\mathscr{B}_{t}}\left(\boldsymbol{\sigma}: \boldsymbol{l}-\rho \dot{e}+\rho \theta \dot{\eta}-\frac{1}{\theta} \boldsymbol{q} \cdot \boldsymbol{\nabla}_{x} \theta\right) d v \geq 0
$$

where $\boldsymbol{\sigma}$ is the Cauchy stress tensor, $\boldsymbol{l}$ is the velocity gradient, $\rho$ is the mass density, $e$ is the internal energy per unit mass, $\theta$ is the temperature, $\eta$ is the entropy per unit mass, and $\boldsymbol{q}$ is the heat flux vector. By assuming homogeneous temperature, i.e., that $\boldsymbol{\nabla}_{x} \theta=\mathbf{0}$, the Clausius-Duhem inequality reduces to the form known as the Clausius-Planck (C-P) inequality

$$
\int_{\mathscr{B}_{t}}(\boldsymbol{\sigma}: \boldsymbol{l}-\rho \dot{e}+\rho \theta \dot{\eta}) d v \geq 0 .
$$

If the Helmholtz free energy per unit mass is introduced in the current configuration, $\Psi=e-\theta \eta$, we may write $-\rho \dot{\Psi}=-\rho \dot{e}+\rho \theta \dot{\eta}+\rho \eta \dot{\theta}$. We write the C-P inequality (noting now the longhand notation for the material time derivative) as

$$
\int_{\mathscr{B}_{t}}(\boldsymbol{\sigma}: \boldsymbol{l}-\rho \dot{\Psi}-\rho \eta \dot{\theta}) d v=\int_{\mathscr{B}_{t}}\left(\boldsymbol{\sigma}: \boldsymbol{l}-\rho \frac{D(\Psi)}{D t}-(\rho \eta \dot{\theta}) d v \geq 0\right.
$$



as,

$$
\int_{\overline{\mathscr{B}}}\left(\boldsymbol{\sigma}: \boldsymbol{l}-\rho \frac{D(\Psi)}{D t}-(\rho \eta) \dot{\theta}\right) J^{e} d \bar{V}=\int_{\overline{\mathscr{B}}}\left(J^{e} \boldsymbol{\sigma}: \boldsymbol{l}-\bar{\rho} \frac{D(\Psi)}{D t}-(\bar{\rho} \eta) \dot{\theta}\right) d \bar{V} \geq 0 .
$$

Furthermore, we say that this must hold in the vicinity of any point $\bar{P} \in \overline{\mathscr{B}}$, i.e., for an arbitrarily small volume element, to obtain the local form of the C-P inequality in $\overline{\mathscr{B}}$, also known as the local plastic dissipation $\overline{\mathcal{D}}_{\text {loc }}$,

$$
\overline{\mathcal{D}}_{l o c}:=J^{e} \boldsymbol{\sigma}: \boldsymbol{l}-\bar{\rho} \frac{D(\Psi)}{D t}-(\bar{\rho} \eta) \dot{\theta} \geq 0 .
$$

If the material changes volume plastically, i.e., $\bar{\rho} \neq \rho_{0}$, it is a simple exercise to show that (making use of Eq. $(5 b))$

$$
\bar{\rho} \frac{D(\Psi)}{D t}=\frac{D(\bar{\rho} \Psi)}{D t}+(\bar{\rho} \Psi) \frac{\dot{J}^{p}}{J^{p}},
$$

where the second term on the $R H S$ of Eq. (11) is an additional contribution to the dissipation from the volumetric plastic deformation. Furthermore, the rate of mechanical work (stress-power) $J^{e} \boldsymbol{\sigma}: \boldsymbol{l}$, appearing in the first term of Eq. (10), can be expanded, first making use of the additive split of the velocity gradient

$$
\begin{aligned}
\boldsymbol{l} & =\boldsymbol{l}^{e}+\boldsymbol{l}^{p} \\
& =\dot{\boldsymbol{F}}^{e} \boldsymbol{F}^{e^{-1}}+\boldsymbol{F}^{e} \overline{\boldsymbol{L}}^{p} \boldsymbol{F}^{e^{-1}},
\end{aligned}
$$

where $\overline{\boldsymbol{L}}^{p}=\dot{\boldsymbol{F}}^{p} \boldsymbol{F}^{p^{-1}}$ is the plastic velocity gradient in $\overline{\mathscr{B}}$. The expression for the stress in the intermediate configuration is given by the second Piola-Kirchhoff stress $\overline{\boldsymbol{S}}$ through a Piola map (Truesdell and Toupin, 1960), defined by

$$
\boldsymbol{\sigma}=\frac{1}{J^{e}} \boldsymbol{F}^{e} \overline{\boldsymbol{S}} \boldsymbol{F}^{e^{T}}, \sigma_{i j}=\frac{1}{J^{e}} F_{i \bar{I}}^{e} \bar{S}_{\bar{I} \bar{J}} F_{j \bar{J}}^{e}
$$

Carrying out the contraction in Eq. (10) gives

$$
\begin{aligned}
\boldsymbol{l}: \boldsymbol{\sigma} J^{e} & =\left(\dot{\boldsymbol{F}}^{e} \boldsymbol{F}^{e}\right): \overline{\boldsymbol{S}}+\overline{\boldsymbol{L}}^{p}:\left(\overline{\boldsymbol{C}}^{e} \overline{\boldsymbol{S}}\right) \\
& =\frac{1}{2} \dot{\overline{\boldsymbol{C}}}^{e}: \overline{\boldsymbol{S}}+\overline{\boldsymbol{L}}^{p}: \overline{\boldsymbol{M}}
\end{aligned}
$$

where we have introduced the unsymmetric Mandel stress $\overline{\boldsymbol{M}}:=\overline{\boldsymbol{C}^{e}} \overline{\boldsymbol{S}}$ (Mandel, 1974) defined according to the elastic right Cauchy-Green deformation tensor $\overline{\boldsymbol{C}}^{e}=\boldsymbol{F}^{e^{T}} \boldsymbol{F}^{e}$ acting on $\overline{\boldsymbol{S}}$. The dissipation inequality can hence be written as

$$
\overline{\mathcal{D}}_{l o c}=\frac{1}{2} \dot{\overline{\boldsymbol{C}}}^{e}: \overline{\boldsymbol{S}}+\overline{\boldsymbol{L}}^{p}: \overline{\boldsymbol{M}}-\frac{D(\bar{\rho} \Psi)}{D t}-(\bar{\rho} \Psi) \frac{\dot{J}^{p}}{J^{p}}-(\bar{\rho} \eta) \dot{\theta} \geq 0 .
$$

The free energy per unit plastically deformed volume $(\bar{\rho} \Psi)$ is postulated with respect to the elasticallyunloaded intermediate configuration. It is assumed to be a function of the state of the material, allowing for the introduction of internal variables associated with the dissipation mechanisms. The present work makes use of the simplifying assumption that the deformation processes are isothermal, allowing for uncoupling of the deformation from changes in temperature. Further, the free energy is assumed to depend on the elastic stored energy function $\overline{\mathcal{W}}\left(\overline{\boldsymbol{C}}^{e}\right)$, which is also known as the per unit plastically deformed volume strain energy density, the uncoupled hardening potential $\overline{\mathcal{H}}\left(\bar{Z}_{\alpha}\right)$, and a thermal potential $\bar{G}(\theta)$, where we have introduced the $n_{\alpha}$ scalar strain-like internal state variables associated with isotropic hardening $\bar{Z}_{\alpha}$ for $\alpha=1$ to $n_{\alpha}$ (in Section 4, two isotropic hardening variables will be introduced for a specific Drucker-Prager type model, but for now the number of ISV's is left general). A further generalization would be to introduce tensor valued internal state variables associated with kinematic hardening and the evolution of texture; however, we will restrict the discussion to isotropic hardening relevant to geomaterials in what follows. We note also that 
for the general case of material anisotropy, second order projection tensors would need to be introduced into the stored energy function in order to allow it to be expressed as an isotropic function representing an present intermediate configuration formulation will be restricted to the case of material isotropy (keeping in mind that it allows for the possibility of anisotropy). Accordingly, the free energy density in $\overline{\mathscr{B}}$ takes the form

$$
\bar{\rho} \Psi=\bar{\rho} \hat{\Psi}\left(\overline{\boldsymbol{C}}^{e}, \bar{Z}_{\alpha}, \theta\right)=\overline{\mathcal{W}}\left(\overline{\boldsymbol{C}}^{e}\right)+\overline{\mathcal{H}}(\bar{Z})+\bar{G}(\theta) .
$$

Such that

$$
\frac{D(\bar{\rho} \Psi)}{D t}=\frac{\partial(\bar{\rho} \Psi)}{\partial \overline{\boldsymbol{C}}^{e}}: \dot{\overline{\boldsymbol{C}}}^{e}+\sum_{\alpha=1}^{n_{\alpha}} \frac{\partial(\bar{\rho} \Psi)}{\partial \bar{Z}_{\alpha}} \dot{\bar{Z}}_{\alpha}+\frac{\partial(\bar{\rho} \Psi)}{\partial \theta} \dot{\theta}
$$

Combining Eqs. (15) and (17) provides,

$$
\begin{aligned}
\overline{\mathcal{D}}_{l o c}=\left(\frac{1}{2} \overline{\boldsymbol{S}}-\right. & \left.\frac{\partial(\bar{\rho} \Psi)}{\partial \overline{\boldsymbol{C}}^{e}}\right): \dot{\overline{\boldsymbol{C}}}^{e}+\overline{\boldsymbol{M}}: \overline{\boldsymbol{L}}^{p}-(\bar{\rho} \Psi) \frac{\dot{J}^{p}}{J^{p}} \\
& -\left((\bar{\rho} \eta)+\frac{\partial(\bar{\rho} \Psi)}{\partial \theta}\right) \dot{\theta}-\sum_{\alpha=1}^{n_{\alpha}} \frac{\partial(\bar{\rho} \Psi)}{\partial \bar{Z}_{\alpha}} \dot{\bar{Z}}_{\alpha} \geq 0
\end{aligned}
$$

Following the standard arguments of what has become to be known as the "Coleman and Noll method" (Coleman and Noll, 1963; Coleman and Gurtin, 1967), Eq. (18) must hold for any arbitrary elastic deformation (hence $\forall \dot{\bar{C}}^{e}$ ) and temperature $(\dot{\theta})$, such that the first and fourth terms on the RHS of Eq.(18) must vanish, yielding the Second-Piola Kirchhoff stress and entropy density in $\overline{\mathscr{B}}$ as,

$$
\begin{aligned}
\overline{\boldsymbol{S}} & =2 \frac{\partial(\bar{\rho} \Psi)}{\partial \overline{\boldsymbol{C}}^{e}}, \\
(\bar{\rho} \eta) & =-\frac{\partial(\bar{\rho} \Psi)}{\partial \theta} .
\end{aligned}
$$
variables $\bar{Z}_{\alpha}$,

$$
\bar{\beta}_{\alpha}:=\frac{\partial(\bar{\rho} \Psi)}{\partial \bar{Z}_{\alpha}} .
$$

239 The plastic dissipation in $\overline{\mathscr{B}}$ can then be written as

$$
\overline{\mathcal{D}}_{l o c}=\overline{\boldsymbol{M}}: \overline{\boldsymbol{L}}^{p}-(\bar{\rho} \Psi) \frac{\dot{J}^{p}}{J^{p}}-\sum_{\alpha=1}^{n_{\alpha}} \bar{\beta}_{\alpha}: \dot{\bar{Z}}_{\alpha} \geq 0 .
$$

240 Recall that $J^{p}:=\operatorname{det}\left[\boldsymbol{F}^{p}\right]$, hence $\dot{J}^{p}=J^{p} \boldsymbol{F}^{p^{-T}}: \dot{\boldsymbol{F}}^{p}$ and

$$
\frac{j^{p}}{J^{p}}=\boldsymbol{F}^{p^{-T}}: \dot{\boldsymbol{F}}^{p}, \quad \frac{\dot{J}^{p}}{J^{p}}=F_{I \bar{I}}^{p^{-1}} \dot{F}_{\bar{I}}^{p} .
$$

${ }^{241}$ Also recall that $\bar{L}_{\bar{I} \bar{J}}^{p}:=\dot{F}_{\bar{I} I}^{p} F_{I \bar{J}}^{p^{-1}}$, such that the contribution to the dissipation from the volumetric plastic ${ }_{242}$ deformation is the trace of the plastic velocity gradient $\operatorname{tr}\left[\overline{\boldsymbol{L}}^{p}\right]=\overline{\mathbf{1}}: \overline{\boldsymbol{L}}^{p}$, i.e.,

$$
\bar{\delta}_{\bar{I} \bar{J}} \bar{L}_{\bar{I} \bar{J}}=\delta_{\bar{I} \bar{J}} \dot{F}_{\bar{I} I}^{p} F_{I \bar{J}}^{p^{-1}}=F_{I \bar{I}}^{p^{-1}} \dot{F}_{\bar{I} I}^{p}=\frac{\dot{J}^{p}}{J^{p}} .
$$


Then the plastic dissipative terms energy conjugate to $\overline{\boldsymbol{L}}^{p}$ which occur in Eq. (21) can be written as

$$
\begin{aligned}
\overline{\boldsymbol{M}}: \overline{\boldsymbol{L}}^{p}-(\bar{\rho} \Psi) \frac{\dot{J}^{p}}{J^{p}} & =[\overline{\boldsymbol{M}}-(\bar{\rho} \Psi) \overline{\mathbf{1}}]: \overline{\boldsymbol{L}}^{p} \\
& =\overline{\boldsymbol{\Xi}}: \overline{\boldsymbol{L}}^{p}
\end{aligned}
$$

where we have defined $\bar{\Xi}:=\overline{\boldsymbol{M}}-(\bar{\rho} \Psi) \overline{\mathbf{1}}$, which we refer to as the Eshelby-Mandel stress, and is recognized as (the negative of) the Eshelby stress in $\overline{\mathscr{B}}$ (Eshelby, 1975): the stress measure that is energy conjugate to $\overline{\boldsymbol{L}}^{p}$ in Eq. (21). The dissipation inequality then finally takes the form

$$
\overline{\mathcal{D}}_{l o c}=\bar{\Xi}: \overline{\mathbf{L}}^{p}-\sum_{\alpha=1}^{n_{\alpha}} \bar{\beta}_{\alpha} \dot{\bar{Z}}_{\alpha} \geq 0
$$

The flow rule will then be defined using $\bar{\Xi}$. Accordingly, the plastic potential function $\overline{\mathcal{G}}$ is then taken to be of the functional form

$$
\overline{\mathcal{G}}:=\hat{\overline{\mathcal{G}}}\left(\bar{\Xi}, \bar{\beta}_{\alpha}\right) .
$$

It remains to determine the canonical form of the evolution equations. In associative plasticity, it is common to invoke the principle of maximum plastic dissipation (PMPD) (von Mises, 1928; Hill, 1948; Mandel, 1964) in order to develop the canonical forms of the evolution equations. The formal application of the PMPD in associative elastoplasticity has been well established in the literature (cf. Lubliner, 1984, 1986; Simo, 1988; Hackl, 1997; Simo and Hughes, 1998), and applications of the PMPD involving Eshelby-like stresses include the works of Rakotomanana (2004) and Miehe and Gürses (2007). However, based on experimental evidence, it is oftentimes customary to relax the PMPD for geomaterials by assuming existence of a plastic potential function $\overline{\mathcal{G}}$ that differs from the yield function $\overline{\mathcal{F}}$, i.e., $\overline{\mathcal{G}} \neq \overline{\mathcal{F}}$, which frames the constitutive model within non-associative plasticity (Vermeer and de Borst, 1984). It is important to note that relaxing the PMPD is not synonymous with violating the second law of thermodynamics (in the form of the C-D inequality, or C-P inequality), i.e., the second law may still be satisfied with suitable selection of plastic potential function $\overline{\mathcal{G}}$ (usually similar functional form as the yield function $\overline{\mathcal{F}}$, but with different material parameters). The dissipation inequality of Eq. (25) can be computed for each increment of deformation to ensure that it is not violated (which will be done in Section 5 for the numerical simulations presented there). We then pose the flow rule without requiring satisfaction of the PMPD,

$$
\begin{aligned}
& \overline{\boldsymbol{L}}^{p}=\dot{\bar{\gamma}} \frac{\partial \overline{\mathcal{G}}}{\partial \overline{\bar{\Xi}}}, \\
& \dot{\bar{Z}}_{\alpha}=-\dot{\bar{\gamma}} \frac{\partial \overline{\mathcal{G}}}{\partial \bar{\beta}_{\alpha}},
\end{aligned}
$$

where $\bar{\gamma}$ is the plastic multiplier.

The solution of $\dot{\boldsymbol{F}}^{p}$ is obtained by noting the definition of $\overline{\boldsymbol{L}}^{p}=\dot{\boldsymbol{F}}^{p} \boldsymbol{F}^{p^{-1}}$, such that Eq. (27a) can then be written as

$$
\dot{\boldsymbol{F}}^{p}=\dot{\bar{\gamma}} \frac{\partial \overline{\mathcal{G}}}{\partial \overline{\mathbf{\Xi}}} \boldsymbol{F}^{p}
$$

The yield function is then proposed to be of a functional form similar to that of the plastic potential function,

$$
\overline{\mathcal{F}}=\hat{\overline{\mathcal{F}}}\left(\bar{\Xi}, \bar{\beta}_{\alpha}\right),
$$

and the Karush-Kuhn-Tucker (KKT) and consistency conditions are given in the standard way (cf. Borja, 2013) by

$$
\dot{\bar{\gamma}} \geq 0, \quad \overline{\mathcal{F}} \leq 0, \quad \dot{\bar{\gamma}} \overline{\mathcal{F}}=0, \quad \dot{\bar{\gamma}} \dot{\overline{\mathcal{F}}}=0
$$

Further discussion on the choice of yield and plastic potential functions is given in Section 4, where specific 
functions are proposed and complete expressions of the constitutive and evolution equations are presented, along with their numerical implementation.

\subsection{Representation in the current configuration}

Here we derive the spatial form of the constitutive equations, i.e., in the current configuration $\mathscr{B}_{\text {t }}$, showing that the spatial Eshelby-Cauchy stress tensor is found to be energy conjugate to the velocity gradient when finite plastic volume change is considered. The C-P inequality is here first enforced with respect to the current configuration volume, which requires the free energy to be posed per unit volume thereof. The following sub-sections (Section 3.3 and 3.4) provide algorithmically convenient alternative formulations that pose the free energy per reference unit volume (both of the material and intermediate configurations, respectively) such that moduli of the free energy function can be thus defined.

The Clausius-Planck inequality is a constraint that must hold at any time $t$, such that

$$
\int_{\mathscr{B}_{t}}(\boldsymbol{l}: \boldsymbol{\sigma}-\rho \dot{\Psi}-(\rho \eta) \dot{\theta}) d v \geq 0 .
$$

Similarly, as was shown in Section 3.1, for non-constant mass density (due to deformation, not mass exchange) we find,

$$
\begin{aligned}
\rho \dot{\Psi} & =\frac{D(\rho \Psi)}{D t}+(\rho \Psi) \frac{\dot{J}}{J} \\
& =\frac{D(\rho \Psi)}{D t}+(\rho \Psi) \mathbf{1}: \boldsymbol{l} .
\end{aligned}
$$

285 The Clausius-Planck inequality can hence be written as

$$
\begin{aligned}
& \int_{\mathscr{B}_{t}}\left[\boldsymbol{l}: \boldsymbol{\sigma}-\frac{D(\rho \Psi)}{D t}-(\rho \Psi) \mathbf{1}: \boldsymbol{l}-(\rho \eta) \dot{\theta}\right] \\
& =\int_{\mathscr{B}_{t}}\left[(\boldsymbol{\sigma}-(\rho \Psi) \mathbf{1}): \boldsymbol{l}-\frac{D(\rho \Psi)}{D t}-(\rho \eta) \dot{\theta}\right] \\
& =\int_{\mathscr{B}_{t}}\left[\boldsymbol{\xi}: \boldsymbol{l}-\frac{D(\rho \Psi)}{D t}-(\rho \eta) \dot{\theta}\right] d v \geq 0,
\end{aligned}
$$

where we define the Eshelby-Cauchy stress tensor $\boldsymbol{\xi}$ that is energy-conjugate to the velocity gradient $\boldsymbol{l}$,

$$
\boldsymbol{\xi}:=\boldsymbol{\sigma}-(\rho \Psi) \mathbf{1}, \quad \xi_{i j}:=\sigma_{i j}-(\rho \Psi) \delta_{i j} .
$$

Because of the assumption of elastic material isotropy, the free energy per unit mass can be taken to be a function of the spatial elastic left Cauchy-Green tensor $\boldsymbol{b}^{e}$ (cf. Simo, 1998), and we choose now a single scalar strain-like internal state variable $z$ associated with isotropic hardening/softening (which will be sufficient for our specific choice of Modified Cam-Clay material model that will be presented in Section 4.2 for the spatial representation), such that

$$
\rho \Psi=\rho \hat{\Psi}\left(\boldsymbol{b}^{e}, z, \theta\right),
$$

giving

$$
\frac{D(\rho \Psi)}{D t}=\frac{\partial(\rho \Psi)}{\partial \boldsymbol{b}^{e}}: \dot{\boldsymbol{b}}^{e}+\frac{\partial(\rho \Psi)}{\partial z} \dot{z}+\frac{\partial(\rho \Psi)}{\partial \theta} \dot{\theta} .
$$

Recalling the additive split $\boldsymbol{l}=\boldsymbol{l}^{e}+\boldsymbol{l}^{p}$ and using that the symmetry of $\boldsymbol{b}^{e}$ allows for the expression

$$
\frac{\partial(\rho \Psi)}{\partial \boldsymbol{b}^{e}}: \dot{\boldsymbol{b}}^{e}=2 \frac{\partial(\rho \Psi)}{\partial \boldsymbol{b}^{e}} \boldsymbol{b}^{e}: \boldsymbol{l}^{e},
$$


the Clausius-Planck inequality can now be written as

$$
\int_{\mathscr{B}_{t}}\left[\left(\boldsymbol{\xi}-2 \frac{\partial(\rho \Psi)}{\partial \boldsymbol{b}^{e}} \boldsymbol{b}^{e}\right): \boldsymbol{l}^{e}+\boldsymbol{\xi}: \boldsymbol{l}^{p}-\frac{\partial(\rho \Psi)}{\partial z} \dot{z}-\left((\rho \eta)+\frac{\partial(\rho \Psi)}{\partial \theta}\right) \dot{\theta}\right] d v \geq 0 .
$$

This provides the constitutive equations for the Eshelby-Cauchy stress $\boldsymbol{\xi}$, energy-conjugate to $\boldsymbol{l}^{e}$, the entropy density $(\rho \eta)$, and the corresponding thermodynamical force $\beta$ energy-conjugate to $\dot{z}$ :

$$
\begin{aligned}
\boldsymbol{\xi} & =2 \frac{\partial(\rho \Psi)}{\partial \boldsymbol{b}^{e}} \boldsymbol{b}^{e}, \\
(\rho \eta) & =-\frac{\partial(\rho \Psi)}{\partial \theta}, \\
\beta & :=\frac{\partial(\rho \Psi)}{\partial z} .
\end{aligned}
$$

Decomposing the plastic velocity gradient into symmetric and skew parts $\boldsymbol{l}^{p}=\boldsymbol{d}^{p}+\boldsymbol{w}^{p}$, where $\operatorname{sym}\left[\boldsymbol{l}^{p}\right]=\boldsymbol{d}^{p}$ and skew $\left[\boldsymbol{l}^{p}\right]=\boldsymbol{w}^{p}$, the further simplification can be made that the Eshelby-Cauchy stress is energy conjugate to the plastic deformation rate $\boldsymbol{d}^{p}$ since symmetry of $\boldsymbol{\xi}$ leads to $\boldsymbol{\xi}: \boldsymbol{w}^{p}=0$. The plastic dissipation inequality has hence been obtained, expressing it in the local form in $\mathscr{B}_{t}$, we now must have that

$$
\mathcal{D}_{l o c}:=\boldsymbol{\xi}: \boldsymbol{d}^{p}-\beta \dot{z} \geq 0 .
$$

The plastic potential and yield functions are introduced allowing for non-associative plasticity analogously to as done in the intermediate configuration representation, such that $\mathcal{G} \neq \mathcal{F}$ (see Section 3.1 for detailed discussion), respectively,

$$
\mathcal{G}:=\hat{\mathcal{G}}(\boldsymbol{\xi}, \beta), \quad \mathcal{F}:=\hat{\mathcal{F}}(\boldsymbol{\xi}, \beta) .
$$

Providing the flow rule:

$$
\begin{aligned}
& \boldsymbol{d}^{p}=\dot{\gamma} \frac{\partial \mathcal{G}}{\partial \boldsymbol{\xi}}, \\
& \dot{z}=-\dot{\gamma} \frac{\partial \mathcal{G}}{\partial \beta},
\end{aligned}
$$

and the KKT and consistency conditions are enforced with respect to the yield function as before.

Note that because of the restriction to material isotropy (elastic and plastic), the orientation of the intermediate configuration is irrelevant and we choose here to assign a value to the plastic spin of $\overline{\boldsymbol{W}}^{p} \equiv \mathbf{0}$ (cf. Simo, 1998). Noting Eq. (12), this implies also that $\boldsymbol{w}^{p} \equiv \mathbf{0}$. This can be seen by making use of the polar decomposition $\boldsymbol{F}^{e}=\boldsymbol{R}^{e} \overline{\boldsymbol{U}}^{e}$, where $\boldsymbol{R}^{e}$ is the orthogonal elastic rotation tensor and $\overline{\boldsymbol{U}}^{e}$ is the symmetric right elastic stretch tensor. The plastic spin of the current configuration is then given by

$$
\begin{aligned}
\boldsymbol{w}^{p} & =\operatorname{skew}\left[\boldsymbol{F}^{e} \overline{\boldsymbol{L}}^{p} \boldsymbol{F}^{e^{-1}}\right]=\operatorname{skew}\left[\boldsymbol{F}^{e} \overline{\boldsymbol{D}}^{p} \boldsymbol{F}^{e^{-1}}\right] \\
& =\frac{1}{2}\left[\boldsymbol{F}^{e} \overline{\boldsymbol{D}}^{p} \boldsymbol{F}^{e^{-1}}-\boldsymbol{F}^{e^{-T}} \overline{\boldsymbol{D}}^{p^{T}} \boldsymbol{F}^{e^{T}}\right] \\
& =\frac{1}{2} \boldsymbol{R}^{e}\left[\overline{\boldsymbol{U}}^{e} \overline{\boldsymbol{D}}^{p} \overline{\boldsymbol{U}}^{e^{-1}}-\overline{\boldsymbol{U}}^{e^{-T}} \overline{\boldsymbol{D}}^{p^{T}} \overline{\boldsymbol{U}}^{e^{T}}\right] \boldsymbol{R}^{e^{T}} \\
& =\frac{1}{2} \boldsymbol{R}^{e}\left[\overline{\boldsymbol{D}}^{p}-\overline{\boldsymbol{D}}^{p^{T}}\right] \boldsymbol{R}^{e^{T}} \\
& =\mathbf{0}
\end{aligned}
$$

311 where it was used that $\overline{\boldsymbol{U}}^{e}$ and $\overline{\boldsymbol{D}}^{p}$ are symmetric and coaxial tensors, and therefore commute. The plastic 
deformation rates in the current and intermediate configurations are hence related by

$$
\boldsymbol{d}^{p}=\boldsymbol{F}^{e} \overline{\boldsymbol{D}}^{p} \boldsymbol{F}^{e^{-1}}
$$

which furnishes the relation between the plastic part of the deformation gradient and the deformation rate in the current configuration (recalling that $\overline{\boldsymbol{D}}^{p}=\dot{\boldsymbol{F}}^{p} \boldsymbol{F}^{p^{-1}}$ ).

\subsection{Spatial representation with respect to material referential unit volume}

Let us briefly derive the "standard" implementation with respect to the current configuration for deformation variables $\boldsymbol{b}^{e}$ and $z$ (i.e., assuming material isotropy) and material reference configuration for the free energy $\left(\rho_{0} \Psi\right)$ (cf. de Souza Neto et al., 2008; Borja, 2013), in order that we may compare and contrast these equations with those derived in terms of the Eshelby-Mandel stress $\overline{\boldsymbol{\Xi}}$ (intermediate configuration) and Eshelby-Cauchy stress $\boldsymbol{\xi}$ (current configuration). It is important to emphasize that what we call the "standard" spatial representation here typically makes use of the assumption that the material is not changing volume plastically, which allows the free energy per unit volume to be equitably posed with respect to unit volumes of either the reference or intermediate configurations. Because we are concerned herein with materials that do undergo plastic volume change, this "standard" implementation implies that either plastic volume changes induce changes in stress (thus violating the basic premise that the intermediate configuration is stress-free), or is inconvenient with respect to the algorithmic implementation because the material parameters cannot remain constant in time, as will be discussed in greater detail in Section 4.

The Clausius-Planck inequality can be written as (comparing with Eq.(33)),

$$
\begin{array}{r}
\int_{\mathscr{B}}(\boldsymbol{l}: \boldsymbol{\sigma}-\rho \dot{\Psi}-(\rho \eta) \dot{\theta}) d v \geq 0 \\
\int_{\mathscr{B}_{0}}\left(\boldsymbol{l}: \boldsymbol{\tau}-\frac{D\left(\rho_{0} \Psi\right)}{D t}-\left(\rho_{0} \eta\right) \dot{\theta}\right) d V \\
\int_{\mathscr{B}_{0}}\left[\left(\boldsymbol{\tau}-2 \frac{\partial\left(\rho_{0} \Psi\right)}{\partial \boldsymbol{b}^{e}} \boldsymbol{b}^{e}\right): \boldsymbol{d}^{e}+\boldsymbol{\tau}: \boldsymbol{d}^{p}-\frac{\partial\left(\rho_{0} \Psi\right)}{\partial z} \dot{z}-\left(\left(\rho_{0} \eta\right)+\frac{\partial\left(\rho_{0} \Psi\right)}{\partial \theta}\right) \dot{\theta}\right] d V \geq 0 .
\end{array}
$$

where the single-phase mass balance $D \rho_{0} / D t=0$ was used, along with the symmetry of $\boldsymbol{\tau}$ providing that $\tau: \boldsymbol{w}^{p}=0$. The Kirchhoff stress, entropy density, and Kirchhoff-stress-like ISV in $\mathscr{B}_{0}$ are then written,

$$
\boldsymbol{\tau}=2 \frac{\partial\left(\rho_{0} \Psi\right)}{\partial \boldsymbol{b}^{e}} \boldsymbol{b}^{e}, \quad\left(\rho_{0} \eta\right)=-\frac{\partial\left(\rho_{0} \Psi\right)}{\partial \theta}, \quad \beta^{\tau}:=\frac{\partial\left(\rho_{0} \Psi\right)}{\partial z},
$$

The dissipation inequality in $\mathscr{B}_{0}$ is then expressed as,

$$
\mathcal{D}_{l o c \mid \mathscr{B}_{0}}:=\boldsymbol{\tau}: \boldsymbol{d}^{p}-\beta^{\tau} \dot{z} \geq 0 .
$$

The plastic potential and yield functions are proposed as before, but now in terms of the Kirchhoff stress,

$$
\mathcal{G}^{\tau}:=\hat{\mathcal{G}}\left(\boldsymbol{\tau}, \beta^{\tau}\right), \quad \mathcal{F}^{\tau}:=\hat{\mathcal{F}}\left(\boldsymbol{\tau}, \beta^{\tau}\right) .
$$

The flow and hardening/softening rule is for this case then:

$$
\begin{aligned}
& \boldsymbol{d}^{p}=\dot{\gamma} \frac{\partial \mathcal{G}^{\tau}}{\partial \boldsymbol{\tau}}, \\
& \dot{z}=-\dot{\gamma} \frac{\partial \mathcal{G}^{\tau}}{\partial \beta^{\tau}} .
\end{aligned}
$$

\subsection{Spatial representation with respect to unit volume of the intermediate configuration}

It is, however, of interest to pose the free energy and enforce the C-P inequality with respect to $\overline{\mathscr{B}}$ (not $\mathscr{B}_{0}$ ), since that is truly the "reference" configuration for the constitutive equations under the assumption of 
the multiplicative split of the deformation gradient (as was presented in Section 2 and will be discussed in greater detail in Section 4). Accordingly, we could consider not the Kirchhoff stress $\boldsymbol{\tau}=J \boldsymbol{\sigma}$, but define an alternative stress measure,

$$
\zeta:=J^{e} \boldsymbol{\sigma},
$$

in order that we may pose the C-P inequality with respect to the intermediate configuration and postulate
the free energy in terms of the plastically deformed unit volume. We begin by writing the C-P inequality
with respect to the current configuration expressed in integral and local forms respectively,

$$
\begin{gathered}
\int_{\mathscr{B}}(\boldsymbol{l}: \boldsymbol{\sigma}-\rho \dot{\Psi}-(\rho \eta) \dot{\theta}) d v \geq 0, \\
\mathcal{D}_{l o c}:=\boldsymbol{l}: \boldsymbol{\sigma}-\rho \dot{\Psi}-(\rho \eta) \dot{\theta} \geq 0 .
\end{gathered}
$$

343 Proceeding with the local form, recalling that $\bar{\rho}$ changes in time and making use of the arguments from 344 Section 3.1, the local dissipation inequality can be multiplied through by $J^{e}$ to find

$$
\begin{aligned}
\mathcal{D}_{l o c \mid \overline{\mathscr{B}}} & =\boldsymbol{l}: \boldsymbol{\sigma} J^{e}-J^{e} \rho \frac{D(\Psi)}{D t}-J^{e}(\rho \eta) \dot{\theta} \\
& =\left(\boldsymbol{\zeta}-2 \frac{\partial(\bar{\rho} \Psi)}{\partial \boldsymbol{b}^{e}} \boldsymbol{b}^{e}\right): \boldsymbol{d}^{e}+\boldsymbol{\zeta}: \boldsymbol{d}^{p}-(\bar{\rho} \Psi) \operatorname{tr}\left[\boldsymbol{d}^{p}\right]-\frac{\partial(\bar{\rho} \Psi)}{\partial z} \dot{z}-\left((\bar{\rho} \eta)+\frac{\partial(\bar{\rho} \Psi)}{\partial \theta}\right) \dot{\theta} \\
& =(\boldsymbol{\zeta}-(\bar{\rho} \Psi) \mathbf{1}): \boldsymbol{d}^{p}-\frac{\partial(\bar{\rho} \Psi)}{\partial z} \dot{z} \\
& =\boldsymbol{\xi}^{\zeta}: \boldsymbol{d}^{p}-\frac{\partial(\bar{\rho} \Psi)}{\partial z} \dot{z} \geq 0
\end{aligned}
$$

where we have defined the spatial Eshelby-like stress that we call the "Eshelby-zeta stress",

$$
\boldsymbol{\xi}^{\zeta}:=\zeta-(\bar{\rho} \Psi) \mathbf{1}
$$

which is energy conjugate to the spatial rate of deformation and defined with respect to the free energy per plastically-deformed unit volume. Note that again we used that $\boldsymbol{\xi}^{\zeta}: \boldsymbol{w}^{p}=0$, the standard Coleman-Noll arguments, and Eq. (23), but now also it was used that $\operatorname{tr}\left[\overline{\boldsymbol{L}}^{p}\right]=\operatorname{tr}\left[\boldsymbol{l}^{p}\right]$. The stress, entropy density, and stress-like ISV constitutive equations in $\overline{\mathscr{B}}$ have also been found,

$$
\begin{aligned}
& \boldsymbol{\zeta}=2 \frac{\partial(\bar{\rho} \Psi)}{\partial \boldsymbol{b}^{e}} \boldsymbol{b}^{e}, \\
& (\bar{\rho} \eta)=-\frac{\partial(\bar{\rho} \Psi)}{\partial \theta} \\
& \boldsymbol{\xi}^{\zeta}:=2 \frac{\partial(\bar{\rho} \Psi)}{\partial \boldsymbol{b}^{e}} \boldsymbol{b}^{e}-(\bar{\rho} \Psi) \mathbf{1} \\
& \beta^{\zeta}:=\frac{\partial(\bar{\rho} \Psi)}{\partial z} .
\end{aligned}
$$

350 The local form of the dissipation inequality can now be written,

$$
\mathcal{D}_{l o c \mid \overline{\mathscr{B}}}:=\boldsymbol{\xi}^{\zeta}: \boldsymbol{d}^{p}-\beta^{\zeta} \dot{z} \geq 0
$$

351 The plastic potential and yield functions are now of the form, respectively,

$$
\mathcal{G}^{\xi^{\zeta}}:=\hat{\mathcal{G}}^{\xi^{\zeta}}\left(\boldsymbol{\xi}^{\zeta}, \beta^{\zeta}\right), \quad \mathcal{F}^{\xi^{\zeta}}:=\hat{\mathcal{F}}^{\xi^{\zeta}}\left(\boldsymbol{\xi}^{\zeta}, \beta^{\zeta}\right) .
$$


The flow rule in this case is then given by,

$$
\begin{aligned}
& \boldsymbol{d}^{p}=\dot{\gamma} \frac{\partial \mathcal{G}^{\xi^{\zeta}}}{\partial \boldsymbol{\xi}^{\zeta}}, \\
& \dot{z}=-\dot{\gamma} \frac{\partial \mathcal{G}^{\zeta}}{\partial \beta^{\zeta}} .
\end{aligned}
$$

\section{Specific constitutive equations and their numerical implementation}

Relatively simple classical forms of free energy and corresponding yield and plastic potential functions will be presented so that the various methods presented in Section 3 can be compared: (1) Drucker-Prager (DP) with pressure dependent cap plasticity and Neo-Hookean elasticity after that presented in Regueiro and Ebrahimi (2010), and (2) Modified Cam-Clay (MCC) plasticity and pressure dependent elasticity after that presented in Borja and Tamagnini (1998). The DP criterion will assume a non-associative flow rule and be formulated in the intermediate configuration to compare the Eshelby-Mandel and Mandel formulations. The MCC criterion will have an associated flow rule and be formulated in the current formulation to compare Eshelby-zeta, zeta, and Kirchhoff formulations. The corresponding free energy functions will also be described here in the various forms described in Section 3. The complete formulation of the constitutive equations is presented in terms of the Eshelby-like stresses $\overline{\boldsymbol{\Xi}}$ and $\boldsymbol{\xi}^{\zeta}$, which are the novel formulations that are suggested here for materials undergoing large plastic volumetric deformation.

The Drucker-Prager (DP) and Modified Cam-Clay (MCC) plasticity models are chosen because they are both well established pressure dependent cap-plasticity models, appropriate for modeling the behavior of a broad range of geomaterials. Drucker-Prager plasticity can be related to the classical Mohr-Coulomb friction model describing granular particle contacts (cf. Chang and Bennett, 2015), with cohesion and the angle of internal friction as model parameters. The DP model has been shown to be applicable to a much wider range of materials (beyond just granular), such as concrete (e.g., Jiang and Wu, 2012) and rock (e.g., Bennett et al., 2015), with even such diverse applications as to shape memory alloys (e.g., Kan and Kang, 2010) and to the modeling of bone plasticity (e.g., Feerick and McGarry, 2012). MCC theory has been well established in the context of critical state mechanics, and continues to be developed in the literature (e.g., Borja et al., 2013; Nguyen, 2014), finding diverse application, such as the modeling of powder compaction (Guner et al., 2015), for example. It is important to emphasize that although the MCC and DP models are chosen with geomaterials in mind, they are chosen for the purpose of investigating and demonstrating the implications of using (or not) the Eshelby-like formulations of elastoplasticity for simulations of large plastic volumetric compression. In other words, the intent is not solely to provide a specific constitutive model for a specific material, but rather to provide an example of how the predictions obtained from the various formulations of finite strain elastoplasticity presented in Section 3 compare when implemented with these classical well-proven constitutive models.

The DP and MCC elastoplastic constitutive models are presented in the following as stress update algorithms for the numerical solution of nonlinear initial boundary value problems (IBVP's) in solid mechanics. Specifically, they are designed to provide the constitutive relationship for the iterative solution of the discretized version of the momentum balance equations through the finite element method. Within this context where stress histories are stored at the Gauss integration points, the algorithm consists of a global iteration (typically of the Newton-Raphson type) that determines the nodal displacement vectors iteratively. The deformation gradients are calculated from this nodal displacement vector, which is then used to drive the stress-point integration algorithm at each Gauss point. The stress update algorithms presented in Box 1 and 2 provide the integration algorithm for the local constitutive equations evaluated at each Gauss point and on each global iterate of the incremental IBVP solution. The full details of the finite element implementation are not provided here because examples (presented in Section 6) are restricted to material point simulations, the concern of the current work being the constitutive model. For further details on the standard numerical procedure for finite element calculations within the context of the current work, the reader is referred to Borja (2013). 


\subsection{Drucker-Prager}

After Regueiro and Ebrahimi (2010) the free energy is taken to be of the compressible Neo-Hookean type, with a hardening potential consisting of two scalar strain like ISV's and hardening moduli; however, we include here also the thermal potential:

$$
\begin{aligned}
\bar{\rho} \Psi & :=\overline{\mathcal{W}}\left(\overline{\boldsymbol{C}}^{e}\right)+\overline{\mathcal{H}}\left(\bar{Z}_{1}, \bar{Z}_{2}\right)+\bar{G}\left(\theta_{0}\right), \\
\overline{\mathcal{W}} & :=\frac{\bar{\lambda}}{2}\left(\ln J^{e}\right)^{2}-\bar{\mu} \ln J^{e}+\frac{\bar{\mu}}{2}\left(\operatorname{tr}\left[\overline{\boldsymbol{C}}^{e}\right]-3\right), \\
\overline{\mathcal{H}} & :=\frac{\bar{H}_{1}}{2} \bar{Z}_{1}^{2}+\frac{\bar{H}_{2}}{2}\left(\bar{Z}_{2}^{2}+\frac{\kappa}{\alpha}\left(\exp \left[-\alpha \bar{Z}_{2}\right]-1\right)^{2}\right),
\end{aligned}
$$

where $\bar{\lambda}$ and $\bar{\mu}$ are the Lamé constants, and $\bar{H}_{1}, \bar{H}_{2}, \kappa$ and $\alpha$ are material parameters describing the hardening/softening behavior. When the parameter $\kappa$ is taken as zero, linear hardening in $\bar{Z}_{2}$ is recovered. The choice of linear hardening in $\bar{Z}_{1}$ and exponential in $\bar{Z}_{2}$ is chosen so that the resulting evolutions laws for the associated stress like ISV's will be similar to the form used by Regueiro and Ebrahimi (2010) (although their's was chosen as an ad-hoc hardening rule). The choice of Neo-Hookean elasticity has the advantage of reducing to Hooke's law at small strain. We note that for high pressure modeling, it is sometimes customary to introduce some sort of pressure dependence, such as the Murnaghan equation of state (Murnaghan, 1944), for example; however, in the interest of keeping the present discussion as succinct as possible and keeping the constitutive model consistent with that presented in Regueiro and Ebrahimi (2010), we choose to avoid introducing such complexity here. Similarly, it should be noted that constitutive models developed for certain geomaterials, or even, as often is the case, for certain sets of experimental measurements, often introduce ad-hoc dependence of elastic properties on pressure and/or density state, which is not compatible with having the hardening potential be considered as part of the free energy (cf. Borja and Tamagnini, 1998). We intentionally avoid introducing any such ad-hoc equations here because doing so would be antithetical to our purpose of examining the influence of the Eshelby stress on the constitutive response while maintaining adherence to the thermodynamic principles outlined in Section 3.

The specific forms of the constitutive equations are then found for the various stress measures described in Section 3.2:

$$
\begin{aligned}
& \overline{\boldsymbol{S}}=\bar{\mu} \overline{\mathbf{1}}+\left(\bar{\lambda} \ln J^{e}-\bar{\mu}\right) \overline{\boldsymbol{C}}^{e^{-1}}, \\
& \overline{\boldsymbol{M}}=\bar{\mu} \overline{\boldsymbol{C}}^{e}+\left(\bar{\lambda} \ln J^{e}-\bar{\mu}\right) \overline{\mathbf{1}}, \\
& \overline{\boldsymbol{\Xi}}=\bar{\mu} \overline{\boldsymbol{C}}^{e}+\left(\bar{\lambda} \ln J^{e}-\bar{\mu}-(\bar{\rho} \Psi)\right) \overline{\mathbf{1}},
\end{aligned}
$$

${ }_{418}$

as well as the isotropic hardening stress-like ISV's,

$$
\bar{\beta}_{1}=\bar{H}_{1} \bar{Z}_{1}, \quad \bar{\beta}_{2}=\bar{H}_{2}\left(\bar{Z}_{2}-\kappa\left(\exp \left[-\alpha \bar{Z}_{2}\right]-1\right) \exp \left[-\alpha \bar{Z}_{2}\right]\right) .
$$

The Drucker-Prager (DP) yield function $\overline{\mathcal{F}}$ is taken to be of the form presented by Regueiro and Ebrahimi 
$(2010)^{1}$, but now as a function of $\overline{\boldsymbol{\Xi}}$ :

$$
\begin{aligned}
& \overline{\mathcal{F}}\left(\bar{\Xi}, \bar{\beta}_{1}, \bar{\beta}_{2}\right):=\|\operatorname{dev}[\bar{\Xi}]\|^{2}-F_{\text {cap }}^{\phi}\left(A^{\phi} \bar{\beta}_{1}-B^{\phi} \bar{p}^{\Xi}\right)^{2} \leq 0, \\
& A^{\phi}:=\frac{2 \sqrt{6} \cos \phi}{3+\eta \sin \phi}, B^{\phi}:=\frac{2 \sqrt{6} \sin \phi}{3+\eta \sin \phi},-1 \leq \eta \leq 1, \\
& F_{\text {cap }}^{\phi}:=1-\left\langle\bar{\beta}_{2}-3 \bar{p}^{\Xi}\right\rangle \frac{\bar{\beta}_{2}-3 \bar{p}^{\Xi}}{\left(\bar{X}^{\phi}-\bar{\beta}_{2}\right)^{2}}, \\
& \left\langle\bar{\beta}_{2}-3 \bar{p}^{\Xi}\right\rangle:=\frac{1}{2}\left[\left|\bar{\beta}_{2}-3 \bar{p}^{\Xi}\right|+\left(\bar{\beta}_{2}-3 \bar{p}^{\Xi}\right)\right], \\
& \bar{X}^{\phi}:=\bar{\beta}_{2}-R\left(A^{\phi} \bar{\beta}_{1}-B^{\phi} \bar{\beta}_{2}\right) .
\end{aligned}
$$

Where $\phi$ and $\bar{\beta}_{1}$ are the friction angle and cohesion, respectively. The internal state variables (ISV's) are $\bar{\beta}_{1}$ and $\bar{\beta}_{2}$, associated with shear and compressive hardening/softening, respectively. The position of the cap along the $3 \bar{p}^{\Xi}$ axis is given by $\bar{X}^{\phi}$ and the Macauley bracket $\langle\cdot\rangle$. The material parameter $R$ controls the ellipticity of the cap, and the shape of the yield surface on the octahedral plane is controlled by $-1 \leq \eta \leq 1$, such that $\eta=1$ and $\eta=-1$ coincide with the intersection of the triaxial extension (TE) and triaxial compression (TC) corners of the Mohr-Coulomb yield surface, respectively. For non-associative plasticity, we have similar functional form for the plastic potential function $\overline{\mathcal{G}}$ as for the yield function; the only difference being that the dilatation angle $\psi$ replaces the friction angle $\phi$ in Eq. (61), such that

$$
\overline{\mathcal{G}}\left(\overline{\boldsymbol{\Xi}}, \bar{\beta}_{1}, \bar{\beta}_{2}\right):=\|\operatorname{dev}[\bar{\Xi}]\|^{2}-F_{\text {cap }}^{\psi}\left(A^{\psi} \bar{\beta}_{1}-B^{\psi} \bar{p}^{\Xi}\right)^{2} .
$$

The flow rule specified in Eq. (27) can now be solved by finding the direction of plastic deformation as

$$
\frac{\partial \overline{\mathcal{G}}}{\partial \overline{\mathbf{\Xi}}}=\operatorname{dev}[\overline{\mathbf{\Xi}}]+\overline{\mathcal{C}}^{\psi} \overline{\mathbf{1}}
$$

430 where

$$
\overline{\mathfrak{C}}^{\psi}:=2\left(A^{\psi} \bar{\beta}_{1}-B^{\psi} \bar{p}^{\Xi}\right)\left[\frac{F_{\text {cap }}^{\psi} B^{\psi}}{3}-\left(A^{\psi} \bar{\beta}_{1}-B^{\psi} \bar{p}^{\Xi}\right) \frac{\left\langle\bar{\beta}_{2}-3 \bar{p}^{\Xi}\right\rangle}{\left(\bar{X}^{\psi}-\bar{\beta}_{2}\right)^{2}}\right] .
$$

The evolution equations for the strain like ISV's are similarly found. Note that

$$
\frac{\partial \overline{\mathcal{G}}}{\partial \bar{\beta}_{1}}=2 A^{\psi}\left(A^{\psi} \bar{\beta}_{1}-B^{\psi} \bar{p}^{\Xi}\right)\left[R\left(A^{\psi} \bar{\beta}_{1}-B^{\psi} \bar{p}^{\Xi}\right)\left\langle\bar{\beta}_{2}-3 \bar{p}^{\Xi}\right\rangle \frac{\bar{\beta}_{2}-3 \bar{p}^{\Xi}}{\left(\bar{X}^{\psi}-\bar{\beta}_{2}\right)^{3}}-F_{\text {cap }}^{\psi}\right],
$$

${ }_{432}$ which means that when the the stress state is not on the cap $\left(3 \bar{p}^{\Xi}>\bar{\beta}_{2}\right)$, Eq. (65) reduces to

$$
\left.\frac{\partial \overline{\mathcal{G}}}{\partial \bar{\beta}_{1}}\right|_{3 \bar{p}^{\Xi} \geq \bar{\beta}_{2}}=-2 A^{\psi}\left(A^{\psi} \bar{\beta}_{1}-B^{\psi} \bar{p}^{\Xi}\right)
$$

The evolution of $\bar{\beta}_{1}$ for stress states on the cap, in contrast, is scaled by

$$
\left.\frac{\partial \bar{G}}{\partial \bar{\beta}_{1}}\right|_{3 \bar{p}^{\Xi}<\bar{\beta}_{2}}=2 A^{\psi}\left(A^{\psi} \bar{\beta}_{1}-B^{\psi} \bar{p}^{\Xi}\right)\left[\left(\frac{\bar{\beta}_{2}-\bar{p}^{\Xi}}{\bar{X}^{\psi}-\bar{\beta}_{2}}\right)^{2}\left(\frac{R\left(A^{\psi} \bar{\beta}_{1}-B^{\psi} \bar{p}^{\Xi}\right)}{\bar{X}^{\psi}-\bar{\beta}_{2}}+1\right)-1\right] .
$$

\footnotetext{
${ }^{1}$ Note that Regueiro and Ebrahimi (2010) used the notation $\bar{c}$ for $\bar{\beta}_{1}, \bar{\kappa}$ for $\bar{\beta}_{2}$, and $\beta$ for $\eta$.
} 
To determine the evolution of $\bar{Z}_{2}$ we find

$$
\frac{\partial \overline{\mathcal{G}}}{\partial \bar{\beta}_{2}}=\left\langle\operatorname{sign}\left(\bar{\beta}_{2}-3 \bar{p}^{\Xi}\right)\right\rangle\left(\frac{A^{\psi} \bar{\beta}_{1}-B^{\psi} \bar{p}^{\Xi}}{\bar{X}^{\psi}-\bar{\beta}_{2}}\right)^{2}\left[\left(\bar{\beta}_{2}-3 \bar{p}^{\Xi}\right)+\left\langle\bar{\beta}_{2}-3 \bar{p}^{\Xi}\right\rangle\left(\frac{2 R B^{\psi}}{\left(\bar{X}^{\psi}-\bar{\beta}_{2}\right)}+1\right)\right] .
$$

Eq. (68) ensures that $\bar{\beta}_{2}$ only evolves when $3 \bar{p}^{\Xi}<\bar{\beta}_{2}$, i.e., when the stress state is on the cap. Hardening will occur when $\bar{X}^{\psi}-\bar{\beta}_{2} \geq-2 R B^{\psi}$, and softening will occur otherwise. Making use of the definition of $\bar{X}^{\psi}$ in Eq. (61e), an equivalent statement is that hardening occurs when $A^{\psi} \bar{\beta}_{1}-B^{\psi} \bar{\beta}_{2} \geq B^{\psi}$, otherwise softening.

\subsubsection{Numerical Implementation of the Drucker-Prager Model}

A semi-implicit integration scheme is used to integrate the evolution equations written in the intermediate configuration (cf. Simo and Ortiz, 1985; Moran et al., 1990). The procedure follows that described in detail by Regueiro and Ebrahimi (2010), who provide further details and discussion. We note that Regueiro and Ebrahimi (2010) made use of the assumption that elastic strains are relatively small compared to the plastic strains; however, such an assumption is not necessary here.

The integrated flow and hardening rules are

$$
\begin{aligned}
& \boldsymbol{F}_{n+1}^{p}=\left[\overline{\mathbf{1}}+\Delta \bar{\gamma}_{n+1}\left(\frac{\partial \overline{\mathcal{G}}}{\partial \overline{\mathbf{\Xi}}}\right)_{n}\right] \boldsymbol{F}_{n}^{p}, \\
& \Delta \bar{Z}_{1}=-\Delta \bar{\gamma}_{n+1}\left(\frac{\partial \overline{\mathcal{G}}}{\partial \bar{\beta}_{1}}\right)_{n}, \\
& \Delta \bar{Z}_{2}=-\Delta \bar{\gamma}_{n+1}\left(\frac{\partial \overline{\mathcal{G}}}{\partial \bar{\beta}_{2}}\right)_{n}
\end{aligned}
$$

which provide the solution to the constitutive equations of Eq. (59) for the strain driven problem (with $\left.\boldsymbol{F}_{n+1}^{e}=\boldsymbol{F}_{n+1} \boldsymbol{F}_{n+1}^{p^{-1}}\right)$. The increment of the plastic multiplier is solved at each time step $t_{n+1}$ with a N-R iteration by enforcing the consistency condition, defining the $\mathrm{N}-\mathrm{R}$ residual $\mathcal{R}:=\overline{\mathcal{F}}=0$. A summary of the integration algorithm for the intermediate configuration representation in terms of the Eshelby-Mandel stress is provided in Box 1, and the equations necessary for finding the consistent tangent of the N-R iteration for each time step are summarized Appendix A.

\subsection{Modified Cam-Clay}

The Modified Cam-Clay (MCC) theory of Borja and Tamagnini (1998) is followed, except that no empirical dependence of elastic variables on density state is introduced, so that it is more comparable with the DP model, and so that the effect of introducing the Eshelby-zeta stress into the constitutive equations is not convoluted by the empirical equations. As Borja and Tamagnini (1998) point out, introducing an empirical Cam-Clay consolidation law prohibits the inclusion of a hardening potential in the free energy.

The free energy is then taken as a function of the left Cauchy-Green deformation tensor $\boldsymbol{b}^{e}$ and a single isotropic hardening variable $z$ as described in Section 3.4. The logarithmic strain tensor is introduced $\varepsilon:=1 / 2 \ln \boldsymbol{b}$, such that it is additively composed of elastic and plastic parts, $\varepsilon=\varepsilon^{e}+\varepsilon^{p}$. The deviatoric part of the elastic logarithmic strain is defined as $e^{e}:=\varepsilon^{e}-1 / 3 \operatorname{tr}\left[\varepsilon^{e}\right] \mathbf{1}$. The plastic hardening potential is also assumed to be part of the free energy in lieu of an ad-hoc hardening rule, analogous to that of the DP model:

$$
\bar{\rho} \Psi:=\frac{\bar{K}}{2}\left(\ln J^{e}\right)^{2}+\bar{\mu}\left\|e^{e}\right\|^{2}+\frac{\bar{H}}{2} z^{2}+\frac{\kappa}{\alpha}(\exp [-\alpha z]-1)^{2}+\bar{G}\left(\theta_{0}\right),
$$

where $\bar{K}$ is the $\log$ bulk modulus, $\bar{\mu}$ is the shear modulus, $\bar{H}$ is the hardening/softening modulus, and $\bar{G}\left(\theta_{0}\right)$ is the thermal potential. Similarly as to the DP model, the material parameters $\alpha$ and $\kappa$ control the exponential hardening, where taking $\kappa=0$ recovers a linear hardening rule. The stress constitutive 
equations are then found according to Eq.'s (54a) and (54c):

$$
\begin{aligned}
& \boldsymbol{\zeta}=2 \bar{\mu} \boldsymbol{e}^{e}+\bar{K} \ln J^{e} \mathbf{1}, \\
& \boldsymbol{\xi}^{\zeta}=2 \bar{\mu} \boldsymbol{e}^{e}+\left(\bar{K} \ln J^{e}-\bar{\rho} \Psi\right) \mathbf{1},
\end{aligned}
$$

467
476

The MCC yield function is given as a function of the Eshelby-zeta stress and a single scalar hardening variable,

$$
\mathcal{F}^{\xi^{\zeta}}\left(\boldsymbol{\xi}^{\zeta}, \beta^{\zeta}\right):=\left(\frac{q^{\xi^{\zeta}}}{m}\right)^{2}+p^{\xi^{\zeta}}\left(p^{\xi^{\zeta}}-p_{c}^{\zeta}\right) \leq 0,
$$

470 where $m$ is a material parameter, $q^{\xi^{\zeta}}:=\sqrt{3 / 2}\left\|\operatorname{dev}\left[\boldsymbol{\xi}^{\boldsymbol{\zeta}}\right]\right\| \equiv q^{\zeta}:=\sqrt{3 / 2}\|\operatorname{dev}[\boldsymbol{\zeta}]\|$, and the scalar state ${ }_{471}$ variable $p_{c}^{\zeta}$, called the preconsolidation pressure, is the isotropic hardening stress-like ISV plus a reference 472 (or initial) value, i.e.,

$$
p_{c}^{\zeta}:=p_{c 0}^{\zeta}+\beta^{\zeta}
$$

473 such that,

$$
\dot{p}_{c}^{\zeta} \equiv \dot{\beta}^{\zeta}
$$

$474 \quad$ Making note of the relations

$$
\frac{\partial q^{\xi^{\zeta}}}{\partial \boldsymbol{\xi}^{\zeta}}=\sqrt{\frac{3}{2}} \frac{\operatorname{dev}[\boldsymbol{\zeta}]}{\|\operatorname{dev}[\boldsymbol{\zeta}]\|}:=\sqrt{\frac{3}{2}} \hat{\boldsymbol{n}}, \quad \frac{\partial p^{\xi^{\zeta}}}{\partial \boldsymbol{\xi}^{\zeta}}=\frac{1}{3} \mathbf{1},
$$

475 the direction of plastic deformation for the associated flow rule is found as

$$
\begin{aligned}
\hat{\boldsymbol{m}}:=\frac{\partial \mathcal{F}^{\zeta}}{\partial \boldsymbol{\xi}^{\zeta}} & =2 \frac{q^{\zeta}}{m^{2}} \frac{\partial q^{\zeta}}{\partial \boldsymbol{\xi}^{\zeta}}+2 p^{\xi^{\zeta}} \frac{\partial p^{\xi^{\zeta}}}{\partial \boldsymbol{\xi}^{\zeta}}-\frac{\partial p^{\xi^{\zeta}}}{\partial \boldsymbol{\xi}^{\zeta}} p_{c}^{\zeta} \\
& =\frac{\sqrt{6} q^{\zeta}}{m^{2}} \hat{\boldsymbol{n}}+\frac{2 p^{\xi^{\zeta}}-p_{c}^{\zeta}}{3} \mathbf{1}
\end{aligned}
$$

and for the evolution of the ISV $z$ we similarly find

$$
\frac{\partial \mathcal{F}^{\zeta}}{\partial \beta \zeta}=-p^{\xi^{\zeta}}
$$

such that the flow and hardening rules according to Eq. (57) then respectively become

$$
\boldsymbol{d}^{p}=\dot{\gamma} \hat{\boldsymbol{m}}, \quad \dot{z}=-\dot{\gamma} p^{\xi^{\zeta}}
$$

\subsubsection{Numerical Implementation of the Modified Cam-Clay Model}

For the spatial representation, a backward Euler fully implicit integration scheme making use of the exponential map (cf. Hirsch and Smale, 1974; Gurtin, 1981; Simo, 1992, among others) is employed to integrate the evolution equations in the current configuration. As noted by Borja and Tamagnini (1998), the assumption of material isotropy and the coaxiality of the plastic and elastic principal stretch increments due to the associated flow rule allows the flow rule of Eq. (42) to be expressed as a plastic corrector equation in the space of $\varepsilon^{e}$. This allows the return mapping algorithm of the classical infinitesimal strain theory to be preserved, as has been noted and explored by many (cf. Simo, 1992; Miehe, 1996; Simo, 1998; de Souza Neto et al., 2008). There appears to be, however, some variability in the literature as to the specific procedures and necessary assumptions, so the precise procedure and specific assumptions employed herein are described 
in detail in what follows.

The constitutive equation for the plastic deformation rate $\boldsymbol{d}^{p}$ given in Eq. (79) is expressed as a spatial quantity. Care must be taken in this regard, recalling the relation between the plastic deformation rates the current and intermediate configurations given in Eq. (44) (where the restriction to material isotropy allowed the assumption that $\overline{\boldsymbol{W}}^{p}=\mathbf{0} \Longrightarrow \boldsymbol{w}^{p}=\mathbf{0}$ ). The polar decomposition $\boldsymbol{F}^{e}=\boldsymbol{R}^{e} \overline{\boldsymbol{U}}^{e}$ is again made use of along with the fact that the symmetric and coaxial tensors $\overline{\boldsymbol{D}}^{p}$ and $\overline{\boldsymbol{U}}^{e}$ commute. The plastic deformation rate defined within the current configuration can then be shown to be equal to the elastically rotated plastic deformation rate by expanding Eq. (44), i.e.,

$$
\begin{aligned}
\boldsymbol{d}^{p} & =\boldsymbol{R}^{e} \overline{\boldsymbol{U}}^{e} \overline{\boldsymbol{D}}^{p} \overline{\boldsymbol{U}}^{e^{-1}} \boldsymbol{R}^{e^{T}} \\
& =\boldsymbol{R}^{e} \overline{\boldsymbol{D}}^{p} \boldsymbol{R}^{e^{T}} \\
& =\boldsymbol{R}^{e}\left(\dot{\boldsymbol{F}}^{p} \boldsymbol{F}^{p^{-1}}\right) \boldsymbol{R}^{e^{T}} .
\end{aligned}
$$

The flow rule of Eq. (57) then becomes

$$
\begin{aligned}
\boldsymbol{R}^{e}\left(\dot{\boldsymbol{F}}^{p} \boldsymbol{F}^{p^{-1}}\right) \boldsymbol{R}^{e^{T}} & =\dot{\gamma} \frac{\partial \mathcal{F}^{\zeta}}{\partial \boldsymbol{\xi}^{\zeta}} \\
\Rightarrow \dot{\boldsymbol{F}}^{p} & =\dot{\gamma} \boldsymbol{R}^{e^{T}} \frac{\partial \mathcal{F}^{\zeta}}{\partial \boldsymbol{\xi}^{\zeta}} \boldsymbol{R}^{e} \boldsymbol{F}^{p} .
\end{aligned}
$$

This is solved making use of the exponential map

$$
\boldsymbol{F}_{n+1}^{p}=\exp \left[\left.(\Delta \gamma) \boldsymbol{R}_{n+1}^{e^{T}} \frac{\partial \mathcal{F}^{\zeta}}{\partial \boldsymbol{\xi}^{\zeta}}\right|_{n+1} \boldsymbol{R}_{n+1}^{e}\right] \boldsymbol{F}_{n}^{p},
$$

or, according to the properties of the tensor exponential given that $\boldsymbol{R}^{e}$ is an orthogonal tensor, equivalently expressed as

$$
\boldsymbol{F}_{n+1}^{p}=\boldsymbol{R}_{n+1}^{e^{T}} \exp \left[\left.\Delta \gamma \frac{\partial \mathcal{F}^{\zeta}}{\partial \boldsymbol{\xi}^{\zeta}}\right|_{n+1}\right] \boldsymbol{R}_{n+1}^{e} \boldsymbol{F}_{n}^{p} .
$$

The definition of the incremental deformation gradient that arises from $\boldsymbol{F}_{n+1}=\boldsymbol{f} \boldsymbol{F}_{n}$ is noted, such that

$$
\boldsymbol{f}=\boldsymbol{F}_{n+1} \boldsymbol{F}_{n}^{-1}=\mathbf{1}+\boldsymbol{\nabla}_{x_{n}}[\Delta \boldsymbol{u}] .
$$

The expression for $\boldsymbol{F}_{n+1}^{e}$ is hence obtained from the multiplicative split, Eq. (83), and the property of the matrix exponential function inverse to be

$$
\begin{aligned}
\boldsymbol{F}_{n+1}^{e} & =\boldsymbol{F}_{n+1} \boldsymbol{F}_{n+1}^{p^{-1}} \\
& =\boldsymbol{f} \boldsymbol{F}_{n}^{e} \boldsymbol{R}_{n+1}^{e^{T}} \exp \left[-\left.\Delta \gamma \frac{\partial \mathcal{F}^{\xi}}{\partial \boldsymbol{\xi}^{\zeta}}\right|_{n+1}\right] \boldsymbol{R}_{n+1}^{e} .
\end{aligned}
$$

The trial value of the elastic deformation gradient is $\boldsymbol{F}_{n+1}^{e, t r}:=\boldsymbol{f} \boldsymbol{F}_{n}^{e}$. Equation (85) can hence be written as 504

$$
\boldsymbol{F}_{n+1}^{e}=\boldsymbol{F}_{n+1}^{e, t r} \boldsymbol{R}_{n+1}^{e^{T}} \exp \left[-\left.\Delta \gamma \frac{\partial \mathcal{F} \xi^{\zeta}}{\partial \boldsymbol{\xi}^{\tau}}\right|_{n+1}\right] \boldsymbol{R}_{n+1}^{e} .
$$

Recall the alternative polar decomposition of the deformation gradient $\boldsymbol{F}_{n+1}^{e}=\mathbf{v}_{n+1}^{e} \boldsymbol{R}_{n+1}^{e}$, where $\mathbf{v}_{n+1}^{e}=$ 
${ }_{507} \quad \boldsymbol{F}_{n+1}^{e, t r}=\mathbf{v}_{n+1}^{e, t r} \boldsymbol{R}_{n+1}^{e, t r}$ and $\boldsymbol{R}_{n+1}^{e, t r}=\boldsymbol{R}_{n+1}^{e}\left(\right.$ since $\left.\overline{\boldsymbol{W}}^{p}=\mathbf{0}\right)$ Eq. (86) can be written as

$$
\mathbf{v}_{n+1}^{e}=\mathbf{v}_{n+1}^{e, t r} \exp \left[-\left.\Delta \gamma \frac{\partial \mathcal{F} \xi^{\zeta}}{\partial \boldsymbol{\xi}^{\zeta}}\right|_{n+1}\right]
$$

508 Taking the natural logarithm of both sides of Eq. (87), the return mapping equation in terms of the loga509 rithmic elastic strain $\left(\varepsilon^{e}=\ln \mathbf{v}^{e}\right)$ is obtained,

$$
\begin{aligned}
\boldsymbol{\varepsilon}_{n+1}^{e} & =\varepsilon_{n+1}^{e, t r}-\left.(\Delta \gamma) \frac{\partial \mathcal{F}}{\partial \boldsymbol{\xi}^{\zeta}}\right|_{n+1} \\
& =\boldsymbol{\varepsilon}_{n+1}^{e, t r}-\Delta \gamma \hat{\boldsymbol{m}}_{n+1} .
\end{aligned}
$$

$$
\begin{aligned}
p_{n+1}^{\zeta} & =\left(\bar{K} \ln J_{n+1}^{e}\right) \\
& =\bar{K}\left(\operatorname{tr}\left[\varepsilon_{n+1}^{e, t r}\right]-\Delta \gamma \operatorname{tr}\left[\hat{\boldsymbol{m}}_{n+1}\right]\right) \\
& =\bar{K}\left(\operatorname{tr}\left[\varepsilon_{n+1}^{e, t r}\right]-\Delta \gamma \frac{\partial \mathcal{F}^{\zeta}}{\partial p^{\xi^{\zeta}}}\right) \\
& =p_{n+1}^{\zeta, t r}-\bar{K} \Delta \gamma\left(2 p_{n+1}^{\zeta}-p_{c, n+1}^{\xi^{\zeta}}\right),
\end{aligned}
$$

${ }_{513}$ where it was used that $\ln J^{e}=\operatorname{tr}\left[\varepsilon^{e}\right]$. The Eshelby-zeta mean stress is then

$$
p_{n+1}^{\xi^{\zeta}}=p_{n+1}^{\zeta}-\left.\bar{\rho} \Psi\right|_{n+1}
$$

The zeta (and Eshelby-zeta) deviator stress is found as

$$
\begin{aligned}
q_{n+1}^{\xi^{\zeta}} \equiv q_{n+1}^{\zeta} & =\sqrt{6} \bar{\mu}\left\|\boldsymbol{e}_{n+1}^{e}\right\| \\
& =\sqrt{6} \bar{\mu}\left\|\boldsymbol{e}_{n+1}^{e, t r}-\Delta \gamma \operatorname{dev}\left[\hat{\boldsymbol{m}}_{n+1}\right]\right\| \\
& =q_{n+1}^{\zeta, t r}-6 \bar{\mu} \Delta \gamma \frac{q_{n+1}^{\zeta}}{m^{2}}
\end{aligned}
$$

${ }_{515}$ The stress like ISV becomes

$$
\beta_{n+1}^{\zeta}=\bar{H}\left(z_{n+1}-\kappa(\exp [-\alpha z]-1) \exp [-\alpha z]\right)
$$

516 where the integrated hardening rule is used:

$$
z_{n+1}=z_{n}+\Delta \gamma p^{\xi^{\zeta}}
$$

517 Note that we can write

$$
p_{c, n+1}^{\zeta}=p_{c 0}^{\zeta}+\bar{H}\left(z_{n+1}-\kappa(\exp [-\alpha z]-1) \exp [-\alpha z]\right)
$$

Eq.'s (90) and (91) provide 2 scalar equations for the stress invariants, rearranging,

$$
q_{n+1}^{\xi^{\zeta}}(\Delta \gamma)=q_{n+1}^{\xi^{\zeta}, t r} /\left(1+\frac{6 \bar{\mu} \Delta \gamma}{m^{2}}\right)
$$




$$
p_{n+1}^{\xi^{\zeta}}(\Delta \gamma)=\frac{p_{n+1}^{\xi^{\zeta}, t r}+\bar{K} \Delta \gamma\left(p_{c 0}^{\zeta}+\bar{H} z_{n}\right)}{1+2 \bar{K} \Delta \gamma-\bar{K} \bar{H}(\Delta \gamma)^{2}} .
$$

${ }_{520}$ The time-integrated consistency condition for plastic loading, $\mathcal{F}_{n+1}^{\xi^{\zeta}}=0$, is employed along with a Newton${ }_{521}$ Raphson iteration to solve for $\Delta \gamma_{n+1}$. The residual is defined as

$$
\mathcal{R}:=\mathcal{F}^{\xi^{\zeta}}(\Delta \gamma)=\left(\frac{q^{\zeta}}{m}\right)^{2}+p^{\xi^{\zeta}}\left(p^{\xi^{\zeta}}-p_{c}^{\zeta}\right)=0 .
$$

${ }_{522}$ A summary of the integration algorithm for the current configuration representation in terms of the Eshelby${ }_{523}$ zeta stress is provided in Box 2, and a summary of the equations necessary for finding the consistent tangent ${ }_{524}$ of the N-R iteration at each time step is provided in Appendix B. 
Box 1. Summary of algorithm for intermediate configuration Drucker-Prager Eshelby-Mandel implementation.

Step 1. Given inc. displacement $\Delta \boldsymbol{u}$ and previous values of $\boldsymbol{F}_{n}, \boldsymbol{F}_{n}^{p},\left(\partial_{\bar{\Xi}} \overline{\mathcal{G}}\right)_{n}, \bar{Z}_{n}^{\alpha},\left(\partial_{\bar{\beta}_{\alpha}} \overline{\mathcal{G}}\right)_{n}$,

Step 2. Compute trial elastic step:

$$
\begin{aligned}
& \boldsymbol{f}_{n+1}=\mathbf{1}+\boldsymbol{\nabla}_{x_{n}}[\Delta \boldsymbol{u}] \\
& \boldsymbol{F}_{n+1}=\boldsymbol{f}_{n+1} \boldsymbol{F}_{n} \\
& \boldsymbol{F}_{n+1}^{e, t r}=\boldsymbol{F}_{n+1} \boldsymbol{F}_{n}^{p^{-1}} \\
& \overline{\boldsymbol{C}}^{e, t r}=\left(\boldsymbol{F}_{n+1}^{e, t r}\right)^{T} \boldsymbol{F}_{n+1}^{e, t r} \\
& \bar{\beta}_{n+1}^{\alpha, t r}=\bar{\beta}_{n}^{\alpha} \\
& \left.\bar{\rho} \Psi\right|_{n+1} ^{t r}:=\bar{\rho} \hat{\Psi}\left(\overline{\boldsymbol{C}}_{n+1}^{e, t r}, \bar{Z}_{n}^{1}, \bar{Z}_{n}^{2}, \theta_{0}\right) \\
& \overline{\boldsymbol{\Xi}}_{n+1}^{t r}:=\hat{\bar{\Xi}}\left(\overline{\boldsymbol{C}}^{e, t r},\left.\bar{\rho} \Psi\right|_{n+1} ^{t r}\right) \\
& \overline{\mathcal{F}}_{n+1}^{t r}:=\hat{\overline{\mathcal{F}}}\left(\overline{\boldsymbol{\Xi}}_{n+1}^{t r}, \bar{\beta}_{n}^{1}, \bar{\beta}_{n}^{2}\right) \\
& { }^{2},{ }^{2}
\end{aligned}
$$

Step 3. Check yielding, and solve for $\Delta \bar{\gamma}_{n+1}$ :

$$
\text { if } \begin{aligned}
& \overline{\mathcal{F}}_{n+1}^{t r} \leq 0 \rightarrow \text { Elastic Step: } \\
& \Delta \bar{\gamma}_{n+1}=0 \\
&(\bullet)_{n+1}=(\bullet)_{n+1}^{t r} \\
& \text { EXIT }
\end{aligned}
$$

Else $\rightarrow$ Plastic Step:

While Error $>$ tolerance

$$
\begin{aligned}
& \mathcal{R}=\hat{\mathcal{R}}\left(\Delta \bar{\gamma}_{n+1}^{k}\right):=\overline{\mathcal{F}}_{n+1}^{t r} \\
& \mathcal{R}^{\prime}=\hat{\mathcal{R}}^{\prime}\left(\Delta \bar{\gamma}_{n+1}^{k}\right) \\
& \Delta \bar{\gamma}_{n+1}^{k+1}=\Delta \bar{\gamma}_{n+1}^{k}-\mathcal{R} / \mathcal{R}^{\prime} \\
& \text { Error }=\left|\mathcal{R} / \mathcal{R}^{\prime}\right| \\
& k=k+1 \\
& \text { Update: } \\
& \boldsymbol{F}_{n+1}^{p}=\hat{\boldsymbol{F}}_{n+1}^{p}\left(\Delta \bar{\gamma}_{n+1}^{k}\right) \\
& \boldsymbol{F}_{n+1}^{e}=\boldsymbol{F}_{n+1} \boldsymbol{F}_{n+1}^{p^{-1}} \\
& \bar{Z}_{n+1}^{\alpha}=\hat{\bar{Z}}_{n+1}^{\alpha}\left(\Delta \bar{\gamma}_{n+1}^{k}\right) \\
& \bar{\beta}_{n+1}^{\alpha}=\hat{\bar{\beta}}_{n+1}^{\alpha}\left(\Delta \bar{\gamma}_{n+1}^{k}\right) \\
& \left.\bar{\rho} \Psi\right|_{n+1}=\bar{\rho} \Psi\left(\Delta \bar{\gamma}_{n+1}^{k}\right) \\
& \bar{\Xi}_{n+1}=\hat{\bar{\Xi}}\left(\Delta \bar{\gamma}_{n+1}^{k}\right)
\end{aligned}
$$

Repeat
Box 2. Summary of algorithm for current configuration Modified Cam-Clay Eshelby-zeta implementation.

Step 1. Given inc. displacement $\Delta \boldsymbol{u}$ and previous values of $\boldsymbol{F}_{n}, \boldsymbol{b}_{n}^{e}, \boldsymbol{\varepsilon}_{n}^{e}, z_{n}, \beta_{n}$,

Step 2. Compute trial elastic step:

$$
\begin{aligned}
& \boldsymbol{f}_{n+1}=\mathbf{1}+\boldsymbol{\nabla}_{x_{n}}[\Delta \boldsymbol{u}] \\
& \boldsymbol{F}_{n+1}=\boldsymbol{f}_{n+1} \boldsymbol{F}_{n} \\
& \boldsymbol{b}_{n+1}^{e, t r}=\boldsymbol{f}_{n+1} \boldsymbol{b}_{n}^{e} \boldsymbol{f}_{n+1}^{T} \\
& \boldsymbol{\varepsilon}_{n+1}^{e, t r}=1 / 2 \ln \boldsymbol{b}_{n+1}^{e, t r} \\
& \beta_{n+1}^{t r}=\beta_{n} \\
& \left.\bar{\rho} \Psi\right|_{n+1} ^{t r}:=\bar{\rho} \hat{\Psi}\left(\boldsymbol{b}_{n+1}^{e, t r}, z_{n}, \theta_{0}\right) \\
& \boldsymbol{\xi}_{n+1}^{\zeta, t r}:=\hat{\boldsymbol{\xi}}^{\zeta}\left(\boldsymbol{b}_{n+1}^{e, t r},\left.\bar{\rho} \Psi\right|_{n+1} ^{t r}\right) \\
& \mathcal{F}_{n+1}^{t r}:=\hat{\mathcal{F}}\left(\boldsymbol{\xi}_{n+1}^{\zeta, t r}, \beta_{n}\right)
\end{aligned}
$$

Step 3. Check yielding, and solve for $\Delta \gamma_{n+1}$ :

$$
\text { if } \begin{aligned}
\mathcal{F}_{n+1}^{t r} \leq 0 & \rightarrow \text { Elastic Step: } \\
\Delta \gamma_{n+1} & =0 \\
(\bullet)_{n+1} & =(\bullet)_{n+1}^{t r} \\
\text { EXIT } &
\end{aligned}
$$

Else $\rightarrow$ Plastic Step:

$$
\text { While Error }>\text { tolerance }
$$

$$
\begin{aligned}
& \mathcal{R}=\hat{\mathcal{R}}\left(\Delta \gamma_{n+1}^{k}\right):=\mathcal{F}_{n+1}^{t r} \\
& \mathcal{R}^{\prime}=\hat{\mathcal{R}}^{\prime}\left(\Delta \gamma_{n+1}^{k}\right) \\
& \Delta \gamma_{n+1}^{k+1}=\Delta \gamma_{n+1}^{k}-\mathcal{R} / \mathcal{R}^{\prime} \\
& \text { Error }=\left|\mathcal{R} / \mathcal{R}^{\prime}\right| \\
& k=k+1 \\
& \text { Update: } \\
& \boldsymbol{\varepsilon}_{n+1}^{e}=\hat{\boldsymbol{\varepsilon}}_{n+1}^{e, t r}\left(\Delta \gamma_{n+1}^{k}\right) \\
& \boldsymbol{b}_{n+1}^{e}=\exp \left[2 \boldsymbol{\varepsilon}_{n+1}^{e}\right] \\
& \beta_{n+1}=\hat{\beta}_{n+1}\left(\Delta \gamma_{n+1}^{k}\right) \\
& \left.\bar{\rho} \Psi\right|_{n+1}=\bar{\rho} \Psi\left(\Delta \gamma_{n+1}^{k}\right) \\
& \boldsymbol{\xi}_{n+1}^{\zeta}=\hat{\boldsymbol{\xi}}^{\zeta}\left(\Delta \gamma_{n+1}^{k}\right)
\end{aligned}
$$

Repeat

EXIT

\subsection{Discussion on choice of free energy function}

Note that we choose to pose the free energy with respect to the elastically-unloaded, plastically-deformed intermediate configuration (i.e., per unit volume thereof), which was convenient for the intermediate configuration in terms of the Eshelby-Mandel stress $\bar{\Xi}$, but required us to introduce the alternative "zeta" stress measure for the current configuration formulation in terms of the Eshelby-zeta stress $\boldsymbol{\xi}^{\zeta}$. This follows from the basic premise of the multiplicative split of the deformation gradient, that the elastic deformation occurs purely between the intermediate and current configurations, such that the intermediate configuration is the one to which we must look to as a reference for the "stress-free" state (in a phenomenological sense) of the material (Lee and Liu, 1967; Lee, 1969; Le and Stumpf, 1993). That this must be the case when large plastic volume strains are considered can be made clear by considering the various functional forms of the free 
energies which were posed in Section 3. The isochoric part of the right and left Cauchy-Green deformation tensors are, respectively, $\overline{\boldsymbol{C}}^{e}:=\left(J^{e}\right)^{-2 / 3} \overline{\boldsymbol{C}}^{e}$ and $\boldsymbol{b}^{e}:=\left(J^{e}\right)^{-2 / 3} \boldsymbol{b}^{e}$. The various free energy forms posed in Section 3 can be written now as additively composed of volumetric and isochoric parts of a compressible Neo-Hookean type:

$$
\begin{aligned}
& \bar{\rho} \Psi\left(\overline{\boldsymbol{C}}^{e}, \bar{Z}\right):=\frac{\bar{K}}{2}\left(\ln J^{e}\right)^{2}+\frac{\bar{\mu}}{2} \operatorname{tr}\left[\overline{\boldsymbol{C}}^{e}\right]+\frac{\bar{H}}{2} \bar{Z}^{2}+\bar{G}\left(\theta_{0}\right), \\
& \rho_{0} \Psi\left(\boldsymbol{b}^{e}, z\right):=\frac{K_{0}}{2}\left(\ln J^{e}\right)^{2}+\frac{\mu_{0}}{2} \operatorname{tr}\left[\boldsymbol{b}^{e}\right]+\frac{H_{0}}{2} z^{2}+G\left(\theta_{0}\right), \\
& \bar{\rho} \Psi\left(\boldsymbol{b}^{e}, z\right):=\frac{\bar{K}}{2}\left(\ln J^{e}\right)^{2}+\frac{\bar{\mu}}{2} \operatorname{tr}\left[\boldsymbol{b}^{e}\right]+\frac{\bar{H}}{2} z^{2}+\bar{G}\left(\theta_{0}\right), \\
& \rho \Psi\left(\boldsymbol{b}^{e}, z\right):=\frac{k}{2}\left(\ln J^{e}\right)^{2}+\frac{\mu}{2} \operatorname{tr}\left[\boldsymbol{b}^{e}\right]+\frac{h}{2} z^{2}+g\left(\theta_{0}\right),
\end{aligned}
$$

where $K_{0}, \bar{K}$, and $k$ are the log bulk moduli with respect to volumes in their respective configurations, $\mu_{0}$, $\bar{\mu}$, and $\mu$ are likewise the shear moduli, and $H_{0}, \bar{H}$, and $h$ are likewise isotropic hardening/softening moduli. Clearly, the moduli are related by the Jacobian maps (from Eq.(5a)),

$$
K_{0}=J^{p} \bar{K}=J k, \quad \mu_{0}=J^{p} \bar{\mu}=J \mu, \quad H_{0}=J^{p} \bar{H}=J h,
$$

54 and likewise the thermal potential,

$$
G\left(\theta_{0}\right)=J^{p} \bar{G}\left(\theta_{0}\right)=J g\left(\theta_{0}\right),
$$

which implies that only those moduli defined with respect to one of the configurations can be taken as constant. The thought experiment provided in Appendix $\mathrm{C}$ shows an example where we must choose those moduli defined with respect to the intermediate configuration unit volume to be constant in time (when we allow that the material may change volume plastically) in order to ensure that the intermediate configuration remains stress-free (in a phenomenological, not a defect-mechanics sense).

We note also here that, without loss of generality, it is at this point assumed that the constant thermal potential $\bar{G}\left(\theta_{0}\right)=0$, such that it does not contribute to $(\bar{\rho} \Psi)$ in calculating an Eshelby-like stress. Otherwise, under constant reference temperature $\theta_{0}$ for which $\bar{G}\left(\theta_{0}\right)$ is a constant, the Eshelby-like stresses presented in the numerical results of Section 5 would be shifted by a constant. For full temperature-dependent constitutive modeling of material behavior for problems in which changing temperature $\theta$ could play a role (such as energy foundations, e.g., Wang et al. (2014), or nuclear waste repositories, e.g., Arson and Gatmiri (2012)), this assumption would not be valid.

\section{Numerical examples and comparisons}

The Eshelby-like stresses we have defined in both the intermediate and current configurations $(\overline{\boldsymbol{\Xi}}$ and $\boldsymbol{\xi}$, respectively), as well as the Eshelby-zeta stress $\boldsymbol{\xi}^{\zeta}$ that we have defined with respect to the intermediate configuration volume, differ from the standard stress measures in those configurations (i.e. $\overline{\boldsymbol{M}}, \boldsymbol{\sigma}$, and $\boldsymbol{\zeta}$, respectively) only as far as their volumetric parts, since it is clear from Eq.'s (24), (34), and (53) that $\operatorname{dev}[\overline{\boldsymbol{\Xi}}] \equiv \operatorname{dev}[\overline{\boldsymbol{M}}], \operatorname{dev}[\boldsymbol{\xi}] \equiv \operatorname{dev}[\boldsymbol{\sigma}]$, and $\operatorname{dev}\left[\boldsymbol{\xi}^{\zeta}\right] \equiv \operatorname{dev}[\boldsymbol{\zeta}]$. While in contrast,

$$
\begin{aligned}
& p^{\bar{\Xi}} \equiv p^{\bar{M}}-(\bar{\rho} \Psi), \\
& p^{\xi} \equiv p^{\sigma}-(\rho \Psi), \\
& p^{\xi^{\zeta}} \equiv p^{\zeta}-(\bar{\rho} \Psi) .
\end{aligned}
$$

In order to examine the significance of posing the constitutive equations in terms of the various Eshelby-like stresses, it is therefore of interest to consider the case of large deformation isotropic compression in which 
no deviatoric stresses are developed (for an isotropic material). In so doing, the intention is to demonstrate the significance of introducing the Eshelby-like stresses into the constitutive equations for those materials capable of undergoing significant plastic volumetric deformation.

Both the DP and MCC models were implemented for simulation of the high pressure isotropic compression measurements on Boulder clay conducted by Mun and McCartney (2015); the details of sample preparation and experimental procedures are discussed in their paper. Measurements were taken to a peak pressure (negative mean Cauchy stress) $\mathrm{p}=-p^{\sigma}$ of approximately $160 \mathrm{MPa}$, corresponding to a total nominal compressive volume strain (negative relative volume change) $-\Delta V / V_{0}=1-J$ of approximately $27 \%$.

The constitutive models derived and presented in terms of the various stress measures, as listed in Table 1, have all been implemented. The DP model was implemented in the intermediate configuration, and the MCC model was implemented in both intermediate and current configurations, where such numerical implementation details follow similarly to the DP model implementation of Section 4.1, and thus are not repeated here. Both models were implemented with both linear and exponential hardening rules $(\kappa=0$ and $\kappa=1$, respectively). "Standard" approaches to the formulation of the constitutive equations in terms of the Mandel $\overline{\boldsymbol{M}}$ and zeta $\zeta$ stresses (which do not account for plastic volume change in satisfying the Clausius-Duhem inequality) and the Kirchhoff stress $\boldsymbol{\tau}$ (which implies additional stresses are developed due to plastic volume changes) have also been implemented for the same loading history, initial conditions, and material parameters so that the results can be compared.

The following figures present the results, showing that the standard formulation of the constitutive equations in terms of $\overline{\boldsymbol{M}}$ or $\boldsymbol{\tau}$ (or $\boldsymbol{\zeta}$ ) differs from the (thermodynamically-consistent) Eshelby-like stress formulations at large plastic volumetric strains, while the intermediate and current configuration representations of the Eshelby-like formulations (Eshelby-Mandel and Eshelby-zeta, respectively) are equivalent. The results show that what we call here "standard" formulations can under-predict the deformation at a given value of mean Cauchy stress $p^{\sigma}$ when large plastic volumetric strain occurs (cf. Figure 8), and may violate the second law of thermodynamics as expressed by the plastic dissipation inequality (derived in Section 3 ) under certain modeling and loading conditions (cf. Figure 10).

Table 1: Formulations of constitutive equations presented and implemented for isotropic compression with large plastic volumetric strain.

\begin{tabular}{cccccc}
\hline Config. & $\begin{array}{c}\text { Formulation } \\
\text { name }\end{array}$ & $\begin{array}{c}\text { Stress } \\
\text { measure }\end{array}$ & $\begin{array}{c}\text { Free } \\
\text { energy }\end{array}$ & $\begin{array}{c}\text { Preserves } \\
\overline{\mathscr{B}} \text { stress-free }\end{array}$ & $\begin{array}{c}\text { Thermodynamically } \\
\text { consistent for } J^{p} \neq 1\end{array}$ \\
\hline \hline \multirow{2}{*}{ Eshelby-Mandel } & $\overline{\boldsymbol{\Xi}}$ & $\bar{\rho} \Psi$ & yes & yes \\
& Mandel & $\overline{\boldsymbol{M}}$ & $\bar{\rho} \Psi$ & yes & no \\
\hline \multirow{2}{*}{$\mathscr{B}_{t}$} & Eshelby-zeta & $\boldsymbol{\xi}$ & $\bar{\rho} \Psi$ & yes & yes \\
& Zeta & $\boldsymbol{\zeta}$ & $\bar{\rho} \Psi$ & yes & no \\
& Kirchhoff & $\boldsymbol{\tau}$ & $\rho_{0} \Psi$ & no & yes \\
\hline
\end{tabular}

\subsection{Drucker-Prager numerical results}

The DP model was used to simulate high pressure isotropic compression tests performed on Boulder clay (Mun and McCartney, 2015) using both exponential and linear hardening rules. Figure 2 compares the simulation results to the measurements, plotting negative mean Cauchy stress (pressure) $\mathrm{p}=-p^{\sigma}$ and nominal compressive volume strain (negative relative volume change) $-\Delta V / V_{0}=1-J$. The model was employed in both (1) an Eshelby-Mandel formulation as described in Sections 3.1 and 4.1, and (2) as a "standard" Mandel formulation, where the yield and plastic potential functions were taken as functions of the Mandel stress, i.e., the standard Mandel formulation is recovered by taking

$$
\overline{\mathcal{F}}(\bar{\Xi}) \rightarrow \overline{\mathcal{F}}(\overline{\boldsymbol{M}}), \quad \overline{\mathcal{G}}(\bar{\Xi}) \rightarrow \overline{\mathcal{G}}(\overline{\boldsymbol{M}}) .
$$


The model parameters were calibrated with the Eshelby-Mandel formulation, and then the standard
Mandel formulation was run with the same model parameters in order to compare the results. The calibrated model parameters are presented in Table 2. The elastic moduli were calibrated to match the slope of the unloading curve (assuming a Poisson's ratio of $\approx 0.375$ to be in the range of typical values for geomaterials). The friction angle was taken as an appropriate value for Boulder clay after Mun and McCartney (2015), and the cohesion and dilatancy angle were chosen as typical (relatively low) values for drained clays. The initial value of the preconsolidation stress $=3 \bar{\beta}_{2(0)}$ was taken as a relatively small value to be consistent with that of a remolded clay. The model parameter $\eta$ was taken as its median value of 0 , and the cap ellipticity parameter $R$ taken as a typical value of 10 after Regueiro and Ebrahimi (2010). The hardening/softening parameters $\bar{H}_{1}, \bar{H}_{2}$, and $\alpha$ were then calibrated through an iterative process for a best fit to the measurements separately for the exponential and linear hardening cases $(\kappa=1$ and $\kappa=0$, respectively).

Figure 2(a) shows that the DP model is able to simulate the high pressure isotropic compression tests reasonably well for both the Eshelby-Mandel and Mandel formulations when the exponential hardening rule is used. The difference between the two formulations is relatively minor, with the standard Mandel formulation predicting a slightly stiffer response than the Eshelby-Mandel, of an additional $4.7 \mathrm{MPa}$ at peak strain of $1-J=27 \%$. Figure 2(b) shows the DP model predictions with the linear hardening rule $(\kappa=0)$, which also predicts a stiffer response of the "standard" formulation of $15.3 \mathrm{MPa}$ at the same peak strain. Clearly, the linear hardening rule is not able to precisely capture the highly non-linear observed behavior; however, the results provide interesting further comparison between the Eshelby-Mandel and Mandel formulations of the DP model. When the plastic volumetric deformation is relatively small, the Eshelby-Mandel and Mandel formulations predict nearly the same results; however, at large plastic volumetric strains, the results diverge from each other. The difference between the formulations is accentuated when the linear hardening rule is used. Figure 3 compares the relative elastic and plastic deformation predicted by the DP EshelbyMandel formulation, comparing the predictions of the linear and exponential hardening rules. Although both hardening rules predict nearly the same total amounts of plastic and elastic deformation, the linear hardening clearly consists of significantly more relative plastic work, calculated as the difference between the total area under the curve and the area under the unloading curve of Figure 2. Interestingly, the linear hardening rule predicts greater plastic dissipation evaluated according to the dissipation inequality of Eq. (25) and reported for the calibrated model predictions in Figure 4.

Figure 5 shows the predicted plastic dissipation for both Eshelby-Mandel and Mandel formulations of the DP model when an associated flow rule is used (i.e, $\psi=\phi=0.576$ ) with linear hardening. The hardening modulus $\bar{H}_{2}$ was decreased to a value of $90 \mathrm{MPa}$ for this test to match exactly the non-associated flow rule prediction of Figure 2(b) with all other model parameters kept the same. Notably, the Mandel formulation predicted dissipation is less than that of the non-associated flow rule, as can be seen by comparing to Figure 4, and the Eshelby-Mandel formulation predicted dissipation drops to a negligible amount when the flow rule becomes associative (greater than zero but less than $10 \times 10^{-7} \mathrm{~Pa}$ ).

\subsection{Modified Cam-Clay numerical results}

The MCC model was also calibrated to the measurements of Mun and McCartney (2015) with both linear and exponential hardening rules. The same elastic moduli were used as for the DP model, using that $\bar{K} \equiv \bar{\lambda}+2 / 3 \bar{\mu}$. The initial value of the MCC preconsolidation parameter was taken to be equivalent to the analogous DP model parameter $p_{c 0} \equiv 3 \bar{\beta}_{2(0)}$. The MCC model parameter $m$ was taken as a typical value of 1. The hardening parameters $\bar{H}$ and $\alpha$ were then calibrated to best fit the measurement data through an iterative process. All MCC model parameters are reported in Table 3.

Figure 6 compares the (spatial) Eshelby-zeta and zeta formulations of the MCC model for both exponential $(\kappa=1)$ and linear $(\kappa=0)$ hardening rules. The "standard" zeta formulation is recovered from the Eshelby-zeta formulation by taking

$$
\mathcal{F}^{\xi}\left(\boldsymbol{\xi}^{\zeta}\right) \rightarrow \mathcal{F}^{\zeta}(\zeta)
$$

The MCC model performs comparably to the DP model, matching the experimental measurements reasonably well for both zeta and Eshelby-zeta formulations when the exponential hardening rule is used. As with the DP model, the "standard" zeta formulation matches the Eshelby-zeta formulation at low strain levels, 
Table 2: DP model parameters calibrated to measurements of Mun and McCartney (2015).

\begin{tabular}{cccc}
\hline Parameter & $\begin{array}{c}\text { Exponential } \\
\text { Hardening }\end{array}$ & $\begin{array}{c}\text { Linear } \\
\text { Hardening }\end{array}$ & Units \\
\hline \hline$\kappa$ & 1 & 0 & $(-)$ \\
$\bar{\lambda}$ & 1.50 & 1.50 & $\mathrm{GPa}$ \\
$\bar{\mu}$ & 0.50 & 0.50 & $\mathrm{GPa}$ \\
$\bar{H}_{1}$ & 0 & 0 & $\mathrm{kPa}$ \\
$\bar{H}_{2}$ & 0.900 & 125 & $\mathrm{MPa}$ \\
$\bar{\beta}_{1(0)}$ & 6.00 & 6.00 & $\mathrm{kPa}$ \\
$\bar{\beta}_{2(0)}$ & -8.00 & -8.00 & $\mathrm{kPa}$ \\
$\eta$ & 0 & 0 & - \\
$R$ & 10 & 10 & - \\
$\alpha$ & 5.0 & - & - \\
$\phi$ & 0.576 & 0.576 & radians \\
$\psi$ & 0.175 & 0.175 & radians \\
\hline
\end{tabular}

but becomes stiffer at large volume strains, with a difference at peak strain $1-J=27 \%$ of $5.6 \mathrm{MPa}$. The linear hardening rule again shows a more pronounced difference than the exponential, with the difference being slightly greater with the MCC model than with the DP of $22.1 \mathrm{MPa}$ at the same peak strain (compare Figures 6(b) and 2(b)).

Figure 7 plots the predicted plastic dissipation according to Eq. (55) for both exponential and linear hardening rules. Again, we see that the linear hardening rule predicts a greater dissipation of energy for the "standard" zeta formulation (notice the difference in the scales of the axes). Remarkably, the associative MCC Eshelby-zeta formulation predicts negligible plastic dissipation, similar to the DP model when it was made associative.

Table 3: MCC model parameters calibrated to measurements of Mun and McCartney (2015).

\begin{tabular}{cccc}
\hline Parameter & $\begin{array}{c}\text { Exponential } \\
\text { Hardening }\end{array}$ & $\begin{array}{c}\text { Linear } \\
\text { Hardening }\end{array}$ & Units \\
\hline \hline$\kappa$ & 1 & 0 & - \\
$\bar{K}, K_{0}$ & 1.83 & 1.83 & $\mathrm{GPa}$ \\
$\bar{H}, H_{0}$ & 0.003 & 765 & $\mathrm{MPa}$ \\
$\bar{p}_{c 0}, p_{c 0}^{\zeta}, p_{c 0}^{\tau}$ & -24.0 & -24.0 & $\mathrm{kPa}$ \\
$\alpha$ & 3.20 & - & - \\
$m$ & 1 & 1 & - \\
\hline
\end{tabular}

The MCC model was also implemented in the intermediate configuration for Mandel and Eshelby-Mandel formulations. A comparison of the results of all the formulation types is given in Figure 8 for a monotonic loading corresponding to the isotropic compression tests of Mun and McCartney (2015) (with the same previously calibrated material parameters). The standard Kirchhoff formulation is also included. Recall the Kirchhoff formulation is thermodynamically-consistent in terms of the Clausius-Duhem inequality, but does not preserve the intermediate configuration as stress-free due to the moduli being assumed constant in the material reference configuration (causing volumetric plastic deformation to contribute to the development of stress). The "standard" Kirchhoff formulation is recovered from the Eshelby-zeta formulation by taking

$$
\mathcal{F}^{\zeta}\left(\boldsymbol{\xi}^{\zeta}\right) \rightarrow \mathcal{F}^{\tau}(\boldsymbol{\tau})
$$

with the Kirchhoff formulation having elastic moduli defined with respect to the reference configuration as previously discussed. As can be seen by Figure 8, the intermediate and current configuration Eshelbylike formulations are equivalent. Likewise, the zeta formulation is the spatial equivalent of the Mandel 
formulation (not the Kirchhoff under plastic volume strain, as is commonly assumed).

Figure 9 plots the Kirchhoff simulation with linear hardening, re-run with moduli scaled by the final change in density predicted by the Eshelby-like simulations, i.e.

$$
\begin{aligned}
& \tilde{K}_{0}=J_{\text {final }}^{p} \bar{K}=0.796 \times 1.83 \mathrm{GPa}=1.46 \mathrm{GPa}, \\
& \tilde{H}_{0}=J_{\text {final }}^{p} \bar{H}=0.796 \times 765 \mathrm{MPa}=609 \mathrm{MPa},
\end{aligned}
$$

where $J_{\text {final }}^{p}=0.796$ is the final plastic part of the Jacobian determinant determined from the Eshelby-like simulations. The Eshelby-zeta and zeta formulations of Figure 8 maintain the original moduli reported in Table 3. The calculation of $\tilde{K}_{0}$ and $\tilde{H}_{0}$ is meant to be analogous to assuming these parameters are variable with deformation, whereas $\bar{K}$ and $\bar{H}$ are assumed constant. It is conceivable to update $\tilde{K}_{0}$ and $\tilde{H}_{0}$ with time as $J^{p}$ varies (although this adds some complexity to the algorithm in enforcing the consistency condition); however, that is not the purpose of this exercise. It is seen in Fig. 9 that the Kirchhoff formulation prediction curve of mean Cauchy stress $p^{\sigma}$ is brought within the Eshelby-zeta and zeta formulation prediction curves when the moduli are scaled, ending at the same final mean Cauchy stress as the zeta formulation. One might argue that with a particular non-linear hardening rule, the Eshelby-zeta and Kirchhoff formulation prediction curves in Fig. 9 could be "fitted together," although with different material parameter values between the two formulations; however, the results would depend on the range of deformation over which the fitting is done, and the intermediate configuration would still not be maintained as stress-free.

Figure 10 shows the MCC model prediction for a (sinusoidal) cycle of strain driven isotropic compression, comparing the Eshelby-zeta and "standard" zeta formulation results (recall these are equivalent to the Eshelby-Mandel and Mandel, respectively). Because of the fact that the MCC model predicts yielding under positive mean stress (without any hardening), yielding occurs on the unload part of the cycle (as soon as the stress becomes positive) in addition to the yielding on the loading portion of the cycle. Under these conditions, the "standard" zeta formulation violates the second law as expressed by the dissipation inequality (Figure 10(f)); whereas, the Eshelby-zeta formulation does not (Figure 10(e)).

\section{Conclusions}

We have shown in detail how what we call the Eshelby-Mandel stress $\overline{\boldsymbol{\Xi}}$ and the Eshelby-zeta stress $\boldsymbol{\xi}^{\zeta}$ are thermodynamically energy-conjugate to the plastic velocity gradient $\overline{\boldsymbol{L}}^{p}$ in the intermediate configuration and the plastic deformation rate $\boldsymbol{d}^{p}$ in the current configuration, respectively, when plastic volume change of mass density is considered. We have exploited this conjugacy to establish a hyper-elastoplastic constitutive framework for materials, such as geomaterials, capable of undergoing large plastic changes in volume. This framework is formulated within the plastically-deformed and elastically-relaxed intermediate configuration which arises from the multiplicative split of the deformation gradient and allows for the possibility of material anisotropy. An alternative current configuration (spatial) formulation of the constitutive equations is presented, allowing for what is called the Eshelby-Cauchy $\boldsymbol{\xi}$ and Eshelby-zeta $\boldsymbol{\xi}^{\zeta}$ stresses that arise to be compared to the Kirchhoff and "zeta" stresses in terms of their thermodynamic definitions, providing insight into the significance of considering the Eshelby-like stresses in the constitutive equations.

Specific forms of classical Drucker-Prager and Modified Cam-Clay elastoplasticity models are presented in intermediate and current configuration formulations, respectively, in order that the Eshelby-like stress formulations can be compared with each other and with "standard" formulations that neglect full consideration of plastic volume change in the thermodynamic formulation (i.e., thermodynamic-conjugacy, and preserving the intermediate configuration as stress-free in a phenomenological sense). All methods (Eshelby-like and "standard") are implemented in the simulation of isotropic compression tests consisting of large volumetric strains and compared with experimental measurements. The results show that the (thermodynamicallyinconsistent) "standard" formulation can under-predict volumetric strain at large mean compressive Cauchy stresses in comparison to the (thermodynamically-consistent) Eshelby-like formulations, and even violate the second law of thermodynamics under certain loading conditions.

We find that for isotropic materials capable of exhibiting large plastic volumetric deformations, whether the Kirchhoff stress or Eshelby stresses are used in the formulation, such differences could be conceivably 
$\mathcal{R}^{\prime}=2 \frac{\partial \operatorname{dev}[\bar{\Xi}]}{\partial \Delta \bar{\gamma}}: \operatorname{dev}[\bar{\Xi}]-\frac{\partial F_{\mathrm{cap}}^{\phi}}{\partial \Delta \bar{\gamma}}\left(A^{\phi} \bar{\beta}_{1}-B^{\phi} \bar{p}^{\Xi}\right)^{2}-F_{\mathrm{cap}}^{\phi} 2\left(A^{\phi} \bar{\beta}_{1}-B^{\phi} \bar{p}^{\Xi}\right)\left(A^{\phi} \frac{\partial \bar{\beta}_{1}}{\partial \Delta \bar{\gamma}}-B^{\phi} \frac{\partial \bar{p}^{\Xi}}{\partial \Delta \bar{\gamma}}\right)$,

"calibrated away" when choosing evolution equations and fitting to experimental data. However, if a material is inherently anisotropic and can exhibit significant volumetric plastic deformations (such as shale, or certain soils in a natural, deposited state, for instance), then the (negative of the) Eshelby stress in the intermediate configuration, assuming a multiplicative decomposition, is the proper thermodynamically-conjugate stress to the plastic velocity gradient, such that resulting constitutive equations are derived thereof.

It is noted that the finite deformation elastoplastic constitutive framework presented here may have further implications beyond thermodynamically-conjugate elastoplasticity. The various phenomena for which the Eshelby stress has been applied to, such as heterogeneity, damage, and fracture (as cited in the introduction), suggests that the Eshelby-like stress constitutive framework presented here may be found to have further implications with respect to these phenomena, especially for those materials capable of undergoing large inelastic volume strain. Presently, we are engaged in research efforts to incorporate the ideas presented here into a general rate-dependent 3D (elastically and plastically) anisotropic constitutive model for geomaterials.

\section{Acknowledgments}

This work is partially supported by the US Department of Energy under Grant No. DEFG02-03ER15454 to Stanford University. KCB is grateful for support from the Department of Defense (DoD) through the National Defense Science \& Engineering Graduate Fellowship (NDSEG) Program. RAR is grateful for support from a UPS Foundation Visiting Associate Professor position in the Department of Civil and Environmental Engineering at Stanford University during the initial stages of this work, and also to ONR MURI grant N00014-11-1-0691. The authors also thank Mr. Woongju Mun and Dr. John S. McCartney of the University of California, San Diego, for providing the experimental data for isotropic compression of Boulder clay at high pressures, and also Mr. Erik Jensen of the University of Colorado Boulder for converting such data for plotting purposes.

\section{Appendix A. Summary of equations for D-P Newton-Raphson iteration tangent}

The tangent for the Newton-Raphson iteration is found (dropping the $n+1$ subscript) as

where,

$$
\frac{\partial \operatorname{dev}[\overline{\boldsymbol{\Xi}}]}{\partial \Delta \bar{\gamma}}=\frac{\partial \overline{\boldsymbol{M}}}{\partial \Delta \bar{\gamma}}-\frac{\partial \bar{p}^{M}}{\partial \Delta \bar{\gamma}} \overline{\mathbf{1}}
$$

with,

$$
\frac{\partial \overline{\boldsymbol{M}}}{\partial \Delta \bar{\gamma}}=\bar{\mu} \frac{\partial \overline{\boldsymbol{C}}^{e}}{\partial \Delta \bar{\gamma}}+\bar{\lambda} \frac{\partial \ln J^{e}}{\partial \Delta \bar{\gamma}} \overline{\mathbf{1}},
$$

$$
\frac{\partial \bar{p}^{M}}{\partial \Delta \bar{\gamma}}=\frac{1}{3} \operatorname{tr}\left[\frac{\partial \overline{\boldsymbol{M}}}{\partial \Delta \bar{\gamma}}\right],
$$

$$
\frac{\partial \overline{\boldsymbol{C}}^{e}}{\partial \Delta \bar{\gamma}}=\frac{\partial \boldsymbol{F}^{e}}{\partial \Delta \bar{\gamma}} \boldsymbol{F}^{e}+\boldsymbol{F}^{e^{T}} \frac{\partial \boldsymbol{F}^{e}}{\partial \Delta \bar{\gamma}},
$$

$$
\frac{\partial \boldsymbol{F}^{e}}{\partial \Delta \bar{\gamma}}=-\boldsymbol{F}^{e}\left(\frac{\partial \overline{\mathcal{G}}}{\partial \overline{\bar{\Xi}}}\right)_{n},
$$

743 and where,

$$
\frac{\partial F_{\text {cap }}^{\phi}}{\partial \Delta \bar{\gamma}}=\frac{2\left\langle\bar{\beta}_{2}-3 \bar{p}^{\Xi}\right\rangle}{\left(\bar{X}^{\phi}-\bar{\beta}_{2}\right)^{2}}[\overline{\mathcal{A}}+\overline{\mathcal{B}}],
$$


744 with

$$
\begin{aligned}
& \overline{\mathcal{A}}:=\frac{\left\langle\bar{\beta}_{2}-3 \bar{p}^{\Xi}\right\rangle}{\bar{X}^{\phi}-\bar{\beta}_{2}} \frac{\partial\left(\bar{X}^{\phi}-\bar{\beta}_{2}\right)}{\partial \Delta \bar{\gamma}}-\frac{\partial \bar{\beta}_{2}}{\partial \Delta \bar{\gamma}}, \\
& \overline{\mathcal{B}}:=3 \frac{\partial \bar{p}^{\Xi}}{\partial \Delta \bar{\gamma}}=3\left(\frac{\partial \bar{p}^{M}}{\partial \Delta \bar{\gamma}}+\frac{\partial \bar{\rho} \Psi}{\partial \Delta \bar{\gamma}}\right), \\
& \frac{\partial \bar{\rho} \Psi}{\partial \Delta \bar{\gamma}}=\frac{\partial \overline{\mathcal{W}}}{\partial \Delta \bar{\gamma}}+\frac{\partial \overline{\mathcal{H}}}{\partial \Delta \bar{\gamma}} \\
& \frac{\partial \overline{\mathcal{W}}}{\partial \Delta \bar{\gamma}}=\bar{\mu}\left(2 \frac{\partial \overline{\boldsymbol{C}}^{e}}{\partial \Delta \bar{\gamma}}: \overline{\mathbf{1}}-\frac{\partial \ln J^{e}}{\partial \Delta \bar{\gamma}}\right)+\lambda \ln J^{e} \frac{\partial \ln J^{e}}{\partial \Delta \bar{\gamma}}, \\
& \frac{\partial \overline{\mathcal{H}}}{\partial \Delta \bar{\gamma}}=-\bar{H}_{1} \bar{Z}_{1} \frac{\partial \overline{\mathcal{G}}}{\partial \bar{\beta}_{1}}-\bar{H}_{2} \bar{Z}_{2} \frac{\partial \overline{\mathcal{G}}}{\partial \bar{\beta}_{2}}+\kappa \bar{H}_{2} \frac{\partial \overline{\mathcal{G}}}{\partial \bar{\beta}_{2}}\left(\exp \left[-\alpha \bar{Z}_{2}\right]-1\right) \exp \left[-\alpha \bar{Z}_{2}\right] \\
& \frac{\partial\left(\bar{X}^{\phi}-\bar{\beta}_{2}\right)}{\partial \Delta \bar{\gamma}}=-R\left(A^{\phi} \frac{\partial \bar{\beta}_{1}}{\partial \Delta \bar{\gamma}}-B^{\phi} \frac{\partial \bar{\beta}_{2}}{\partial \Delta \bar{\gamma}}\right), \\
& \frac{\partial \bar{\beta}_{1}}{\partial \Delta \bar{\gamma}}=-\bar{H}_{1} \frac{\partial \overline{\mathcal{G}}}{\partial \bar{\beta}_{2}} \\
& \frac{\partial \bar{\beta}_{2}}{\partial \Delta \bar{\gamma}}=-\bar{H}_{2} \frac{\partial \overline{\mathcal{G}}}{\partial \bar{\beta}_{2}}\left(1+\kappa \alpha \exp \left[-\alpha \bar{Z}_{2}\right]^{2}+\kappa \alpha\left(\exp \left[-\alpha \bar{Z}_{2}\right]-1\right) \exp [-\alpha \bar{Z}]\right)
\end{aligned}
$$

\section{Appendix B. Summary of equations for MCC Newton-Raphson iteration tangent}

The tangent for the Newton-Raphson iteration is found (dropping the $n+1$ subscript) as

$$
\begin{gathered}
\mathcal{R}^{\prime}=\frac{2 q^{\zeta}}{m^{2}} \frac{\partial q^{\zeta}}{\partial \Delta \gamma}+2 p^{\xi^{\zeta}} \frac{\partial p^{\xi^{\zeta}}}{\partial \Delta \gamma}-\frac{\partial p^{\xi^{\zeta}}}{\partial \Delta \gamma} p_{c}^{\zeta}-p^{\xi^{\zeta}} \frac{\partial p_{c}^{\zeta}}{\partial \Delta \gamma} \\
\frac{\partial q^{\zeta}}{\partial \Delta \gamma}=-q^{\zeta, t r}\left(1+\frac{6 \bar{\mu} \Delta \gamma}{m^{2}}\right)^{-2}\left(\frac{6 \bar{\mu}}{m^{2}}\right) \\
\frac{\partial p^{\xi^{\zeta}}}{\partial \Delta \gamma}=\frac{\bar{K}\left(p_{c 0}^{\xi^{\zeta}}+\bar{H} z_{n}\right)}{1+2 \bar{K} \Delta \gamma-\bar{K} \bar{H}(\Delta \gamma)^{2}}-\frac{p^{\xi^{\zeta}, t r}+\bar{K} \Delta \gamma\left(p_{c 0}^{\zeta}+\bar{H} z_{n}\right)}{\left(1+2 \bar{K} \Delta \gamma-\bar{K} \bar{H}(\Delta \gamma)^{2}\right)^{2}}-\frac{\partial \bar{\rho} \Psi}{\partial \gamma}, \\
\frac{\partial \bar{\rho} \Psi}{\partial \Delta \gamma}=\bar{K} \ln J^{e} \frac{\partial \ln J^{e}}{\partial \Delta \gamma}+2 \bar{\mu} \frac{\partial \boldsymbol{e}^{e}}{\partial \Delta \gamma}: \boldsymbol{e}^{e}+\bar{H} z \frac{\partial z}{\partial \Delta \gamma}, \\
\bar{\partial} \frac{\partial z}{\partial \Delta \gamma}\left(1+\kappa \alpha \exp [-\alpha z]^{2}+\kappa \alpha(\exp [-\alpha z]-1) \exp [-\alpha z]\right) \\
\frac{\partial \ln J^{e}}{\partial \Delta \gamma}=-\operatorname{tr}\left[\hat{\boldsymbol{m}}^{t r}\right] \\
\frac{\partial \boldsymbol{e}^{e}}{\partial \Delta \gamma}=-\operatorname{dev}\left[\hat{\boldsymbol{m}}^{t r}\right] \\
\frac{\partial z}{\partial \Delta \gamma}=p^{\xi^{\zeta}}+\Delta \gamma \frac{\partial p^{\xi^{\zeta}}}{\partial \Delta \gamma}
\end{gathered}
$$$$
754 \text { where }
$$ 


\section{Appendix C. Thought experiment on choice of per unit volume elastic moduli}

Consider for a moment our previous per reference volume form of the free energy further simplified for the case of perfect-plasticity (in the absence of hardening) such that we may write

$$
\rho_{0} \Psi=\frac{K_{0}}{2}\left(\ln J^{e}\right)^{2}+\frac{\mu_{0}}{2} \operatorname{tr}\left[\boldsymbol{b}^{e}\right]+G\left(\theta_{0}\right),
$$

where thermal potential $G\left(\theta_{0}\right)$ is independent of deformation based upon the isothermal assumption. If we were to choose $K_{0}$ and $\mu_{0}$ to be constant in time, an increment of purely volumetric plastic deformation (where $J_{2}^{e} \equiv J_{1}^{e}$ ) with $\Delta J=J_{2}-J_{1}=J_{2}^{p} J_{2}^{e}-J_{1}^{p} J_{1}^{e}=\Delta J^{p} J_{1}^{e}$ (where the subscripts denote the plastic states at times $t=t_{1}, t_{2}$ ) would cause a change in the stress state. For example, consider the definition of the Kirchhoff stress measure in Eq. (46), which would yield no change in $\boldsymbol{\tau}$ over the purely plastic increment, i.e., $\Delta \boldsymbol{\tau}=\boldsymbol{\tau}_{2}-\boldsymbol{\tau}_{1}=\mathbf{0}$. However, the actual corresponding Cauchy stress becomes

$$
\begin{aligned}
\Delta \boldsymbol{\sigma} & =\frac{1}{J_{2}} \boldsymbol{\tau}_{2}-\frac{1}{J_{1}} \boldsymbol{\tau}_{1} \\
& =\frac{J_{1}-J_{2}}{J_{1} J_{2}} \boldsymbol{\tau}_{1} \\
& =-\frac{\Delta J^{p} J_{1}^{e}}{J_{1} J_{2}^{p} J_{1}^{e}} \boldsymbol{\tau}_{1} \\
& =-\frac{\Delta J^{p}}{J_{2}^{p}} \boldsymbol{\sigma}_{1} \\
& =\left(\frac{J_{1}^{p}}{J_{2}^{p}}-1\right) \boldsymbol{\sigma}_{1} .
\end{aligned}
$$

Clearly, this predicts a change in the actual Cauchy stress resulting purely from plastic deformation for the perfectly-plastic case; whereas if $J_{2}^{p} \equiv J_{1}^{p} \Longrightarrow \Delta J^{p} \equiv 0$, this problem does not arise. Conversely, if we choose the intermediate configuration to define our constant moduli, we find $\Delta \boldsymbol{\tau}=\boldsymbol{\tau}_{2}-\boldsymbol{\tau}_{1}=\left(J_{1}^{p}+\right.$ $\left.\Delta J^{p}\right)\left(\bar{K} \ln J_{1}^{e}+\bar{\mu} / 2 \dot{\boldsymbol{b}}_{1}^{e}\right)-J_{1}^{p}\left(\bar{K} \ln J_{2}^{e}+\bar{\mu} / 2 \dot{\boldsymbol{b}}_{2}^{e}\right)=\Delta J^{p}\left(\bar{K} \ln J_{1}^{e}+\bar{\mu} / 2 \dot{\boldsymbol{b}}_{1}^{e}\right)$, recalling that $J_{2}^{e}=J_{1}^{e}$ and $\dot{\boldsymbol{b}}_{2}^{e}=\ddot{\boldsymbol{b}}_{1}^{e}$ for our present thought experiment. The corresponding change to the Cauchy stress is $\Delta \boldsymbol{\sigma}=\mathbf{0}$, namely,

$$
\begin{aligned}
\Delta \boldsymbol{\sigma} & =\boldsymbol{\sigma}_{2}-\boldsymbol{\sigma}_{1} \\
& =\frac{J_{2}^{p}}{J_{2}}\left(\bar{K} \ln J_{2}^{e} \mathbf{1}+\frac{\bar{\mu}}{2} \dot{\boldsymbol{b}}_{2}^{e}\right)-\frac{J_{1}^{p}}{J_{1}}\left(\bar{K} \ln J_{1}^{e} \mathbf{1}+\frac{\bar{\mu}}{2} \boldsymbol{b}_{1}^{e}\right) \\
& =\left(\frac{J_{2}^{p}}{J_{2}^{p} J_{2}^{e}}-\frac{J_{1}^{p}}{J_{1}^{p} J_{1}^{e}}\right)\left(\bar{K} \ln J_{1}^{e} \mathbf{1}+\frac{\bar{\mu}}{2} \boldsymbol{b}^{e}\right) \\
& =\left(\frac{1}{J_{1}^{e}}-\frac{1}{J_{1}^{e}}\right)\left(\bar{K} \ln J_{1}^{e} \mathbf{1}+\frac{\bar{\mu}}{2} \boldsymbol{b}^{\boldsymbol{e}}\right) \\
& =\mathbf{0} .
\end{aligned}
$$

\footnotetext{
This motivates the choice of constant parameters in the intermediate configuration for Eshelby-Mandel and Eshelby-zeta stess formulations, for materials that may experience large volumetric plastic (or inelastic) deformation.
}

\section{References}

Arson, C., Gatmiri, B., 2012. Thermo-hydro-mechanical modeling of damage in unsaturated porous media: Theoretical framework and numerical study of the EDZ. Int. J. Numer. Anal. Meth. Geomech. 36, 272-306.

Asaro, R., 1983. Micromechanics of crystals and polycrystals. Adv. App. Mech. 23, $1-115$.

Balieu, R., Kringos, N., 2015. A new thermodynamical framework for finite strain multiplicative elastoplasticity coupled to anisotropic damage. Int. J. Plasticity 70, $126-150$. 
Bammann, D., 2001. A model of crystal plasticity containing a natural length scale. Mat. Sci. Engr. A309-310, 406-410.

Bennett, K., Berla, L., Nix, W., Borja, R., 2015. Instrumented nanoindentation and 3D mechanistic modeling of a shale at multiple scales. Acta Geotech. 10(1), 1-14.

Bilby, B., Bullough, R., Smith, E., 1955. Continuous distributions of dislocations: a new application of the methods of non-Riemannian geometry. Proc. R. Soc. Lond. A, Math. Phys. Eng. Sci. 231, 263-273.

Borja, R., 2013. Plasticity Modeling \& Computation. Springer-Verlag, Berlin-Heidelberg.

Borja, R., Song, X., Wu, W., 2013. Critical state plasticity. Part VII: Triggering a shear band in variably saturated porous media. Comput. Method Appl. M. 261, 66-82.

Borja, R.I., Tamagnini, C., 1998. Cam-Clay plasticity Part III: Extension of the infintesimal model to include finite strains. Comp. Meth. Appl. Mech. Engng. 155, 73-95.

Brepols, T., Vladimirov, I., Reese, S., 2014. Numerical comparison of isotropic hypo- and hyperelastic-based plasticity models with application to industrial forming processes. Int. J. Plasticity $63,18-48$

Brünig, M., 2004. Eshelby stress tensor in large strain anisotropic damage mechanics. Int. J. Mech. Sci. 46, 1763-1782.

Callari, C., Auricchio, F., Sacco, E., 1998. A finite-strain Cam-clay model in the framework of multiplicative elasto-plasticity. Int. J. Plasticity 14(12), $1155-1187$.

Chang, C., Bennett, K., 2015. Micromechanical modeling for the deformation of sand with non-coaxiality between the stress and material axes. J. Eng. Mech., in-press. doi:10.1061/(ASCE) EM.1943-7889.0000966.

Clausmeyer, T., G unner, A., Tekkaya, A., Levkovitch, V., Svendsen, B., 2014. Modeling and finite element simulation of loading-path-dependent hardening in sheet metals during forming. Int. J. Plasticity 63, $64-93$.

Clayton, J., 2011. Nonlinear mechanics of crystals. Springer.

Clayton, J., Bammann, D., McDowell, D., 2004. Anholonomic configuration spaces and metric tensors in finite elastoplasticity. Int. J. Non. Linear Mech. 39, $1039-1049$.

Clayton, J., Hartley, C.S., McDowell, D., 2014. The missing term in the decomposition of the deformation gradient. Int. J. Plast. 52, $51-76$.

Clayton, J., McDowell, D., Bammann, D., 2006. Modeling dislocations and disclinations with finite micropolar elastoplasticity. Int. J. Plast. 22, $210-256$.

Cleja-Tigoiu, S., Maugin, G., 2000. Eshelby's stress tensors in finite elastoplasticity. Acta. Mech. 139, $231-249$.

Coleman, B., Gurtin, M., 1967. Thermodynamics with internal variables. J. Chem. Phys. 47, 597-613.

Coleman, B., Noll, W., 1963. The thermodynamics of elastic materials with heat conduction and viscosity. Archive for Rational Mechanics and Analysis 13(1), 167-168.

Dormieux, L., Kondo, D., 2013. Strength properties of a Drucker-Prager porous medium reinforced by rigid particles. Int. J. Plasticity 51, 218-240.

Epstein, M., Maugin, G., 1990. The energy-momentum tensor and material uniformity in finite elasticity. Acta. Mech. 83, $127-133$.

Epstein, M., Maugin, G., 2000. Thermomechanics of volumetric growth in uniform bodies. Int. J. Plasticity 16, 951-978.

Eringen, A., 1967. Mechanics of Continua. John Wiley and Sons, Inc., New York, London, Sydney.

Eshelby, J., 1951. The force on an elastic singularity. Phil. Trans. Roy. Soc. Lond. 244, 87-112.

Eshelby, J., 1956. Progress in Solid State Physics. Academic Press. volume 3. chapter The continuum theory of lattice defect. pp. $79-144$.

Eshelby, J., 1975. The elastic energy-momentum tensor. J. Elasticity 5(3-4), 321-335.

Feerick, E., McGarry, J., 2012. Cortical bone failure mechanisms during screw pullout. J. Biomech. 45(9), $1666-1672$.

Gajo, A., 2011. Finite strain hyperelastoplastic modelling of saturated porous media with compressible constituents. Int. J. Solid. Struct. 48(11-12), 1738-1753.

Ganghoffer, J-F., 2010. On Eshelby tensors in the context of the thermodynamics of open systems: Application to volumetric growth. Int. J. Eng. Sci. 48, 2081-2098.

Ganghoffer, J-F., 2013. A kinematically and thermodynamically consistent volumetric growth model based on the stress-free configuration. Int. J. Solid Struct. 50, 3446-3459.

Garikipati, K., Arruda, E., Grosh, K., Narayanan, H., Calve, S., 2004. A continuum treatment of growth in biological tissue: the coupling of mass transport and mechanics. J. Mech. Phys. Solids 52, 1595-1625.

Guner, F., Cora, O., Sofuoglu, H., 2015. Numerical modeling of cold powder compaction using multi particle and continuum media approaches. Powder Technol. 271, 238-247.

Gurtin, M., 1981. Mathematics in Science and Engineering. volume 158 of An Introduction to Continuum Mechanics. Academic Press, NY.

Gurtin, M., 1995. The nature of configurational forces. Arch. Rational Mech. Anal. 131, 67-100.

Hirsch, M., Smale, S.,1974. Differential Equations, Dynamical Systems, and Linear Algebra. Academic Press, Inc., San Diego, CA..

Hackl, K., 1997. Generalized standard media and variational principles in classical and finite strain elastoplasticity. J. Mech. Phys. Solids 45(5), 667-688.

Hashiguchi, K., 1993. Mechanical requirements and structures of cyclic plasticity models. Int. J. Plasticity 9(6), 721-748.

Hashiguchi, K., Yamakawa, Y.,2013. Introduction to Finite Strain Theory For Continuum Elasto-Plasticity. John Wiley and Sons, Inc., West Sussex, UK.

Hill, R., 1948. A variational principle of maximum plastic work in classical plasticity. Quart. J. Mech. Appl. Math. 1, 18-28. Holzapfel, G., 2000. Nonlinear Solid Mechanics. John Wiley and Sons Ltd., West Sussex, England.

Holzapfel, G., Gasser, T., Ogden, R., 2000. A new constitutive framework for arterial wall mechanics and a comparative study of material models. J. Elast. $61,1-48$. 
Jeremić, B., Runesson, K., Sture, S., 2001. Finite deformation analysis of geomaterials. Int. J. Numer. Anal. Meth. Geomech. 25, 809-840.

51 Jiang, J., Wu, Y., 2012. Identification of material parameters for Drucker-Prager plasticity model for FRP confined circular concrete columns. Int. J. Solids Struct. 49, 445-456.

Le, K., Stumpf, H., 1993. Constitutive equations for elastoplastic bodies at finite strain: thermodynamic implementation. Acta Mech. 100, 155-170.

Lee, E., 1969. Elastic-plastic deformation at finite strains. J. Appl. Mech. 36, 1-6.

Lee, J., Li, J., Zhang, Z.,2014. Material force in micromorphic plasticity. Arch. Appl. Mech. 84, 1453-1464.

62 Lee, E., Liu, D., 1967. Finite strain elastic-plastic theory with application to plane wave analysis. J. Appl. Phys. 38, 19-27.

65 Malvern, L., 1969. Introduction to the Mechanics of Continuous Media. Prentice-Hall, Inc., Upper Saddle River, NJ.

866 Mandel, J., 1964. Contribution théorique à l'écrouissage et des lois de l'écoulement plastique, in: Proc. 11th Int. Cong. Appl. Mech., pp. 502-509.

Mandel, J., 1974. Foundation of Continuum Thermodynamics. Macmillan, London. chapter Thermodynamics and plasticity. pp. 283-304.

Marsden, J., Hughes, T., 1983. Mathematical Foundations of Elasticity. Prentice-Hall, Inc., Englewood Cliffs, N.J.

Maugin, G., 2013. Sixty years of configurational mechanics (1950-2010). Mech. Res. Comun. 50, 39-49.

72 Maugin, G., 1994. Eshelby stress in elastoplasticity and fracture. Int. J. Plasticity 10, 393-408.

73augin, G., Epstein, M., 1998. Geometrical material structure of elastoplasticity. Int. J. Plasticity 14, 109-115.

874 Miehe, C., 1996. Exponential map algorithm for stress updates in anisotropic multiplicative elastoplasticity for single crystals. Int. J. Numer. Meth. 39, 3367-3390.

Miehe, C., 1998. Constitutive frame of elastoplasticity at large strains based on the notion of a plastic metric. Int. J. Solid. Struct. 35(30), 3859-3897.

Miehe, C., Gürses, E., 2007. A robust algorithm for configurational-force-driven brittle crack propagation with R-adaptive mesh alignment. Int. J. Numer. Meth. Engng. 72, 127-155.

Menzel, A., Steinmann, P., 2007. On configurational forces in multiplicative elastoplasticity. Int. J. Solids Struct. 44, $4442-4471$. von Mises, R., 1928. Mechanik der plastischen formänderung von krinstallen. Math. Mech. 8, 161-185.

Monchiet, V., Charkaluk, E., Kondo, D., 2014. Macroscopic yield criteria for ductile materials containing spheroidal voids: An eshelby-like velocity fields approach. Mech. Mater. 72, 1-18.

Moran, B., Ortiz, M., Shih, C., 1990. Formulation of implicit finite-element methods for multiplicative finite deformation plasticity. Int. J. Numer. Meth. 29(3), 484-514.

Mosler, J., Bruhns, O., 2009. Towards variational constitutive updates for non-associative plasticity models at finite strain: Models based on a volumetric-deviatoric split. Int. J. Solid. Struct. 46(7-8), 1676-1684.

Mun, W., McCartney, J., 2015. Compression mechanisms of unsaturated clay under high stresses. Can. Geotech. J. , $1676-1684$. Murnaghan, F., 1944. The Compressibility of Media under Extreme Pressures. P. Natl. Acad. Sci. USA, 30, 244-247.

Nemat-Nasser, S., 1983. On finite plastic flow of crystalline solids and geomaterials. Transactions of the ASME 50, 1114-1126.

Nguyen, L., Fatahi, B., Khabbaz, H., 2014. A constitutive model for cemented clays capturing cementation degradation. Int. J. Plasticity $56,1-18$.

Ogden, R., 1984. Nonlinear Elastic Deformations. Chicheste, Ellis Horwood.

Ortiz, M., Pandolfi, A., 2004. A variational Cam-clay theory of plasticity. Comp. Method. Appl. M. 193(27-29), 2645-2666.

Rakotomanana, L., 2004. A Geometric Approach to Thermodynamics of Dissipating Continua. Boston: Birkhäser.

Regueiro, R., Bammann, D., Marin, E., Garikipati, K., 2002. A nonlocal phenomenological anisotropic finite deformation plasticity model accounting for dislocation defects. J. Eng. Mater. Technol. 124, 380-387.

Regueiro, R., Ebrahimi, D., 2010. Implicit dynamic three-dimensional finite element analysis of an inelastic biphasic mixture at finite strain Part 1: Application to a simple geomaterial. Comp. Meth. Appl. M. 199, 2024-2049.

Sansour, C., Karlšaj, I., Sorić, J., 2006. A formulation of anisotropic continuum elastoplasticity at finite strains. Part I: Modelling. Int. J. Plasticity 22, 2346-2365.

Sansour, C., Karlšaj, I., Sorić, J., 2007. On a formulation for anisotropic elastoplasticity at finite strains invariant with respect to the intermediate configuration. J. Mech. Phys. Solids 55, 2406-2426.

Shutof, A., Ihlemann, J., 2014. Analysis of some basic approaches to finite strain elasto-plasticity in view of reference change. Int. J. Plasticity 63, 183-197.

Simo, J., 1985. On the computational significance of the intermediate configuration and hyperelastic stress relations in finite deformation elastoplasticity. Mech. Mater. 4, 439-451.

Simo, J., 1988. A framework for finite strain elastoplasticity based on maximum plastic dissipation and the multiplicative decomposition: Part I. Continuum formulation. Comput. Method Appl. M. 66, 199-219.

Simo, J., 1992. Algorithms for static and dynamic multiplicative plasticity that preserve the classical return mapping schemes of the infinitesimal theory. Comput. Method Appl. M. Engng. 99, 61-112.

Simo, J., 1998. Numerical analysis and simulation of plasticity, in: Ciarlet, P., Lions, J. (Eds.), Handbook of Numerical $913 \quad$ Analysis. Elsevier Science. volume 6, pp. 183-499. 
Simo, J., Hughes, T., 1998. Computational Inelasticity. Springer-Verlag.

Simo, J., Meschke, G., 1993. A new class of algorithms for classical plasticity extended to finite strains. applications to geomaterials. Comput. Mech. 11, 253-278.

917 Simo, J., Ortiz, M., 1985. A unified approach to finite deformation elastoplasticity analysis based on the use of hyperelastic constitutive equations. Comp. Meth. Appl. Mech. Engng. 49, 221-245.

919 de Souza Neto, E., Peric, D., Owen, D., 2008. Computational Methods for Plasticity. Wiley, West Susssex.

Steinmann, P., 1996. Views on multiplicative elastoplasticity and the continuum theory of dislocations. Int. J. Engr. Sci. 34, $1717-1735$

Stumpf, H., Makowski, J., Hackl, K., 2009. Dynamical evolution of fracture process region in ductile materials. Int. J. Plasticity $25(5), 995-1010$.

Svendsen, B., 2001. On the modelling of anisotropic elastic and inelastic material behaviour at large deformation. Int. J. Soilds Struct. 38, 9579-9599.

Svendsen, B., Levkovitch, V., Wang, J.,Reusch, F., Reese, S., 2006. Application of the concept of evolving structure tensors to the modeling of initial and induced anisotropy at large deformation. Comput. Struct. 84, 1077-1085.

Svendsen, B., Neff, P., Menzel, A., 2009. On constitutive and configurational aspects of models for gradient continua with microstructure. Math. Mech. 89, 687-697.

Truesdell, C., Noll, W., 1965. The Non-Linear Field Theories of Mechanics. Handbuch der Physik III/3, Flügge, S. (Ed.), Springer, Berlin, Heidelberg, New York.

Truesdell, C., Toupin, R., 1960. The Classical Field Theories. Volume 3(1). Springer, Berlin.

Vermeer, P., de Borst, R., 1984. Non-associated plasticity for soils, concrete, and rock. Heron 29, 3-64.

Vladimirov, I., Pietryga, M., Reese, S., 2010. Anisotropic finite elastoplasticity with nonlinear kinematic and isotropic hardening and application to sheet metal forming Int. J. Plasticity 26, 659-687.

Wang, W., Regueiro, R., McCartney, J., 2014. Coupled axisymmetric thermo-poro-mechanical finite element analysis of energy foundation centrifuge experiments in partially saturated silt. Geotech. Geol. Eng., 1.

(a)

Fig. 2: Drucker-Prager model calibrated to measurement data of Mun and McCartney (2015), showing both Eshelby-Mandel and Mandel formulations for: (a) Exponential hardening rule $(\kappa=1)$, and (b) linear hardening rule $(\kappa=0)$.

Fig. 3: Relative plastic and elastic deformation predicted by Drucker-Prager Eshelby-Mandel formulation, comparing exponential and linear hardening model predictions.

Fig. 4: Plastic dissipation predictions of Drucker-Prager model when calibrated to measurement data of Mun and McCartney (2015) showing both Eshelby-Mandel and Mandel formulations: (a) Exponential hardening rule $(\kappa=1)$, and (b) linear hardening rule $(\kappa=0)$.

Fig. 5: Plastic dissipation predicted by DP with associated flow rule $(\psi=\phi=0.576)$ and linear hardening.

Fig. 6: Modified Cam-Clay model calibrated to measurement data of Mun and McCartney (2015), showing both Eshelby-zeta and zeta formulations for: (a) Exponential hardening rule $(\kappa=1)$, and (b) linear hardening rule $(\kappa=0)$. 
Fig. 7: Plastic dissipation predicted by the Modified Cam-Clay model when calibrated to measurement data of Mun and McCartney (2015), showing both Eshelby-zeta and zeta formulations: (a) Exponential hardening rule $(\kappa=1)$, and (b) linear hardening rule $(\kappa=0)$.

(a)

(b)

Fig. 8: Comparison of MCC model predictions of mean Cauchy stress $p^{\sigma}$ comparing all (a) current configuration formulations, and (b) intermediate configuration formulations.

Fig. 9: Comparison of (current configuration) Kirchhoff, Eshelby-zeta, and zeta formulations with moduli scaled by $J_{\text {final }}^{p}$ for Kirchhoff (scaled) formulation.
(a) Load cycle applied
(b) Pressure
(c) Preconsolidation stress
(d) Free energy
(e) Dissipation: Eshelby-zeta formulation
(f) Dissipation: zeta formulation

Fig. 10: MCC model sinusoidal load/unload cycle comparing Eshelby-zeta and zeta formulations, and showing the zeta formulation (and therefore also the Mandel formulation) violates the dissipation inequality in this case. 
Figure

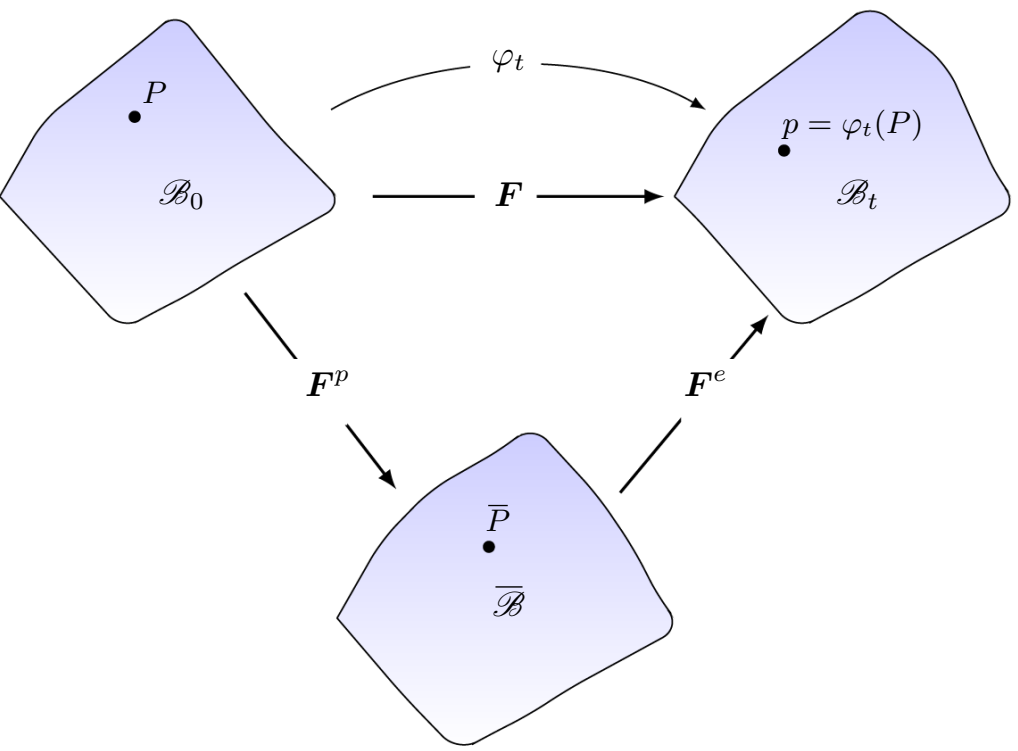


Figure $\cdot 10^{8}$

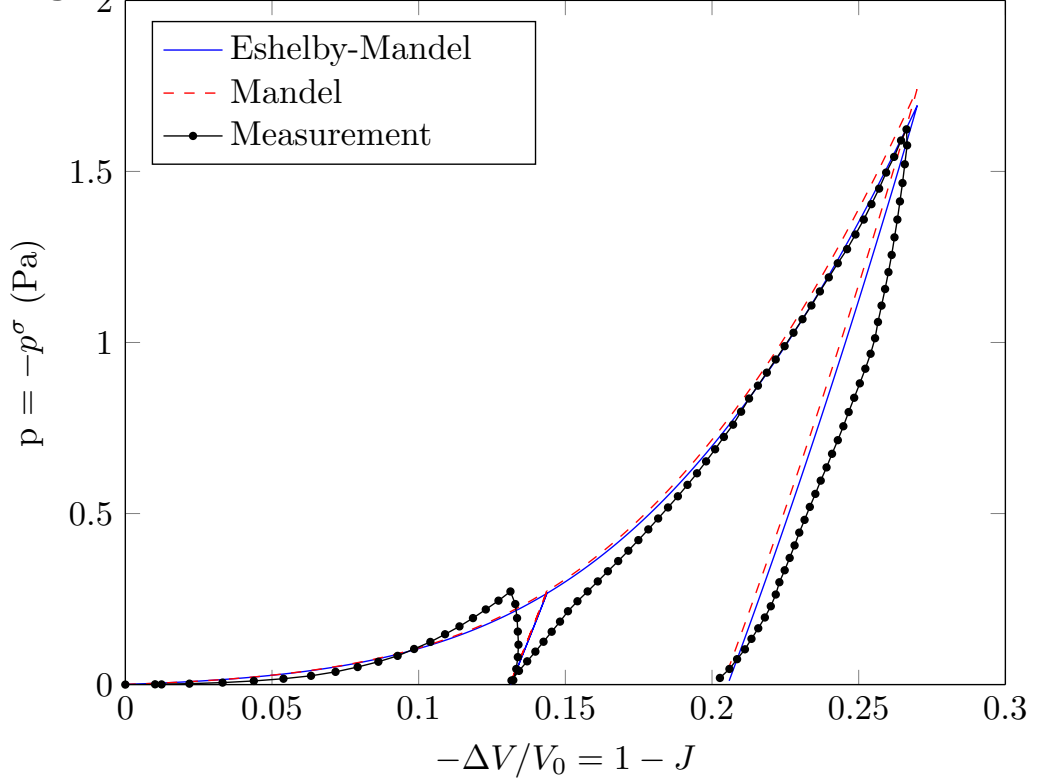


Figure $\cdot 10^{8}$

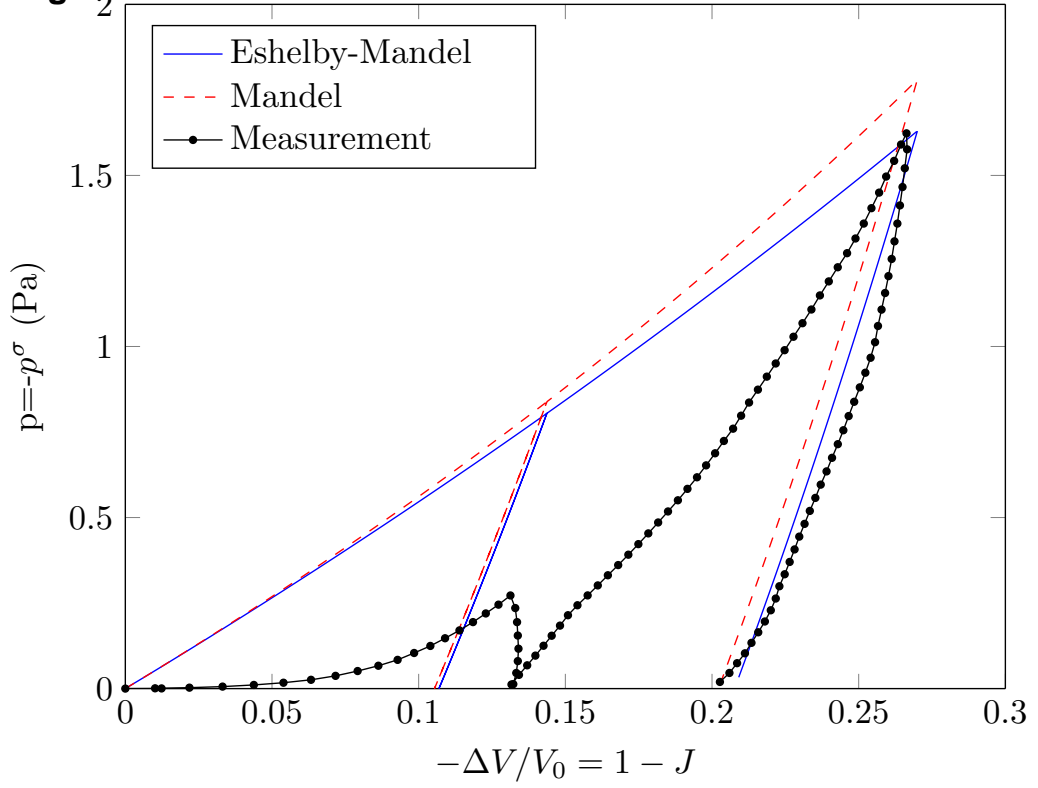




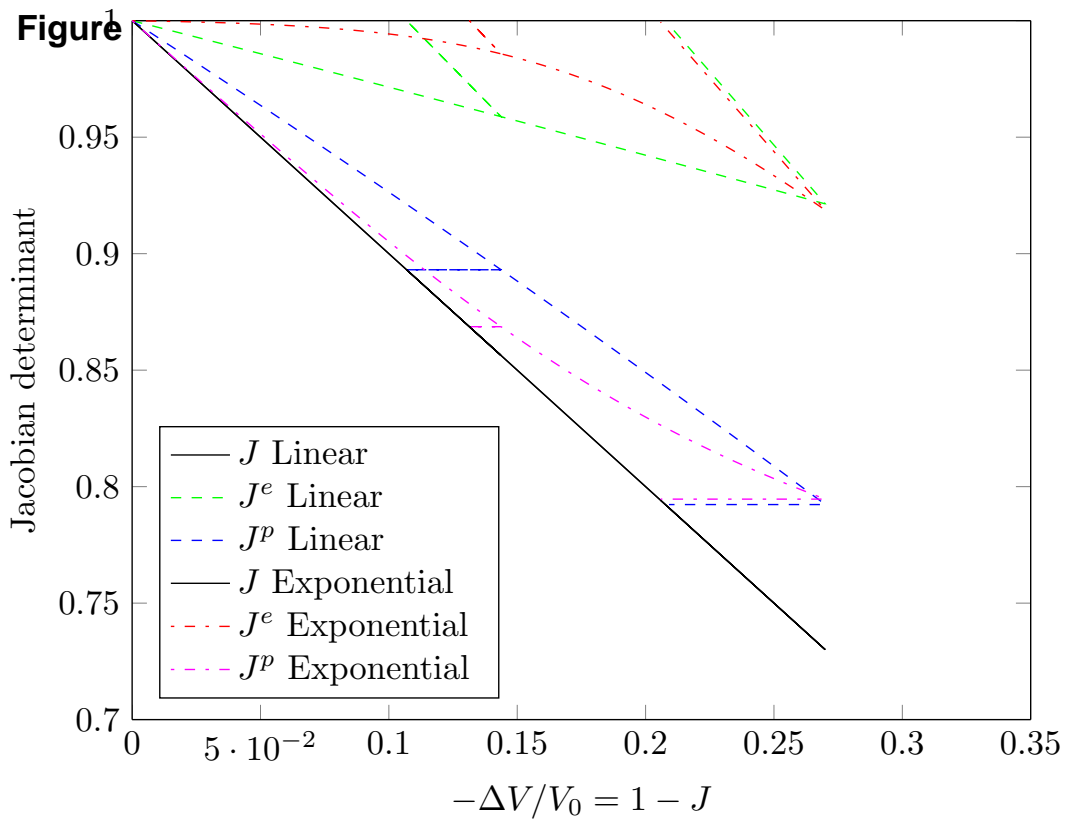


Figựe

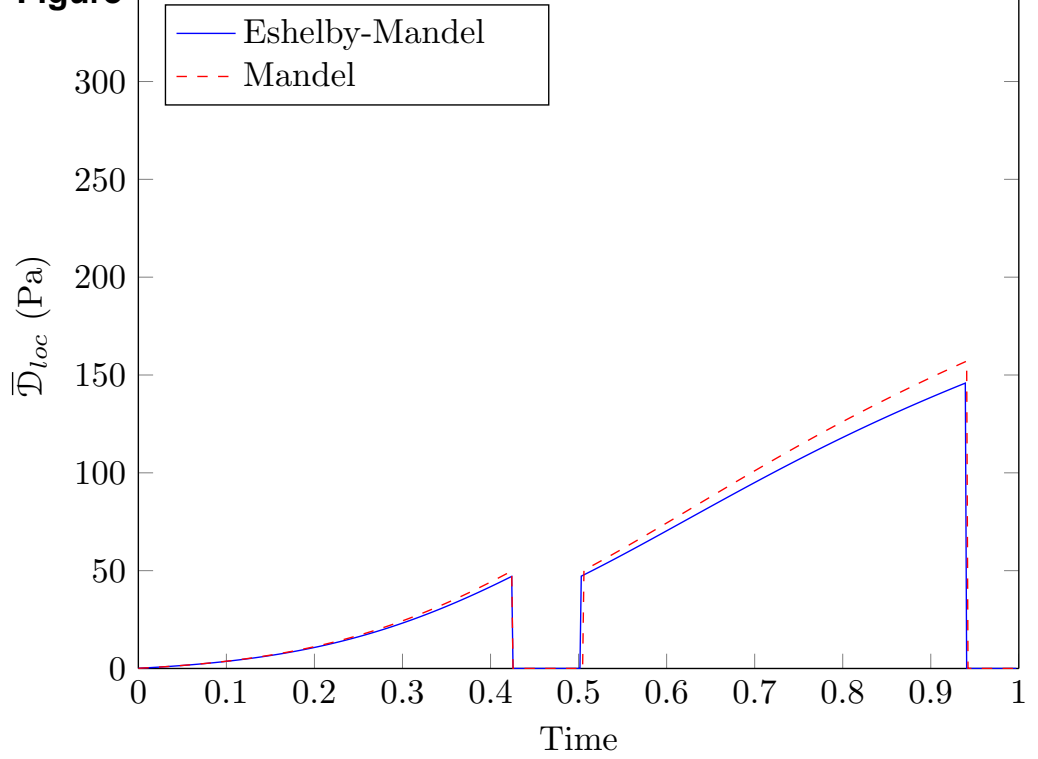




\section{Figựẹ}

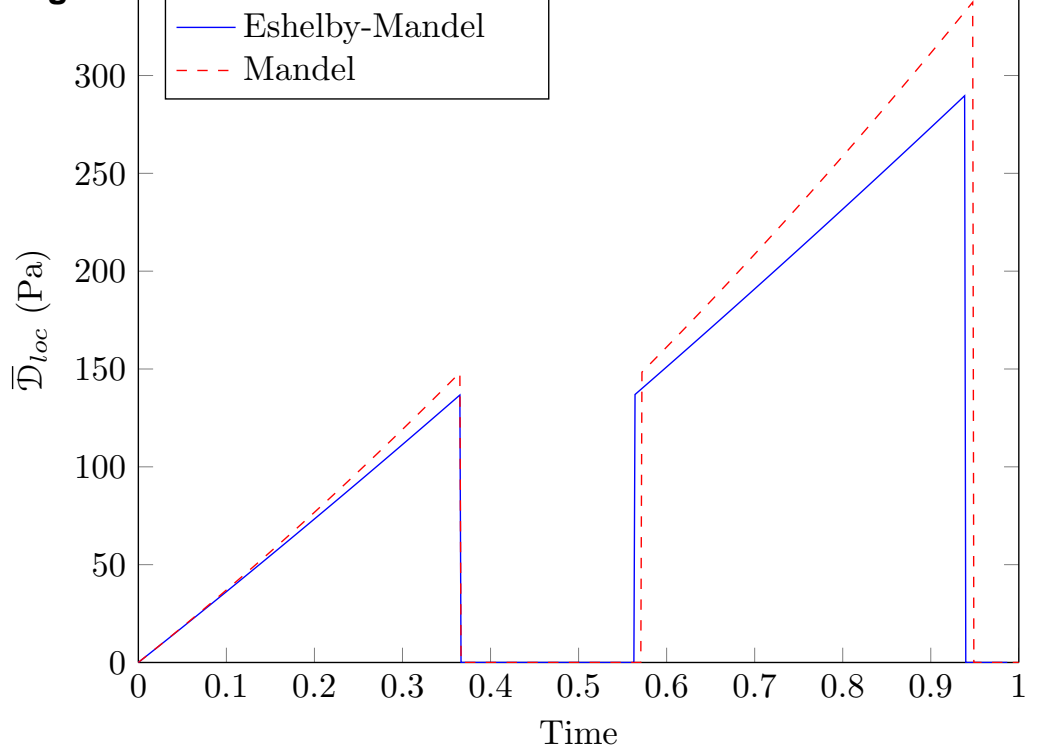




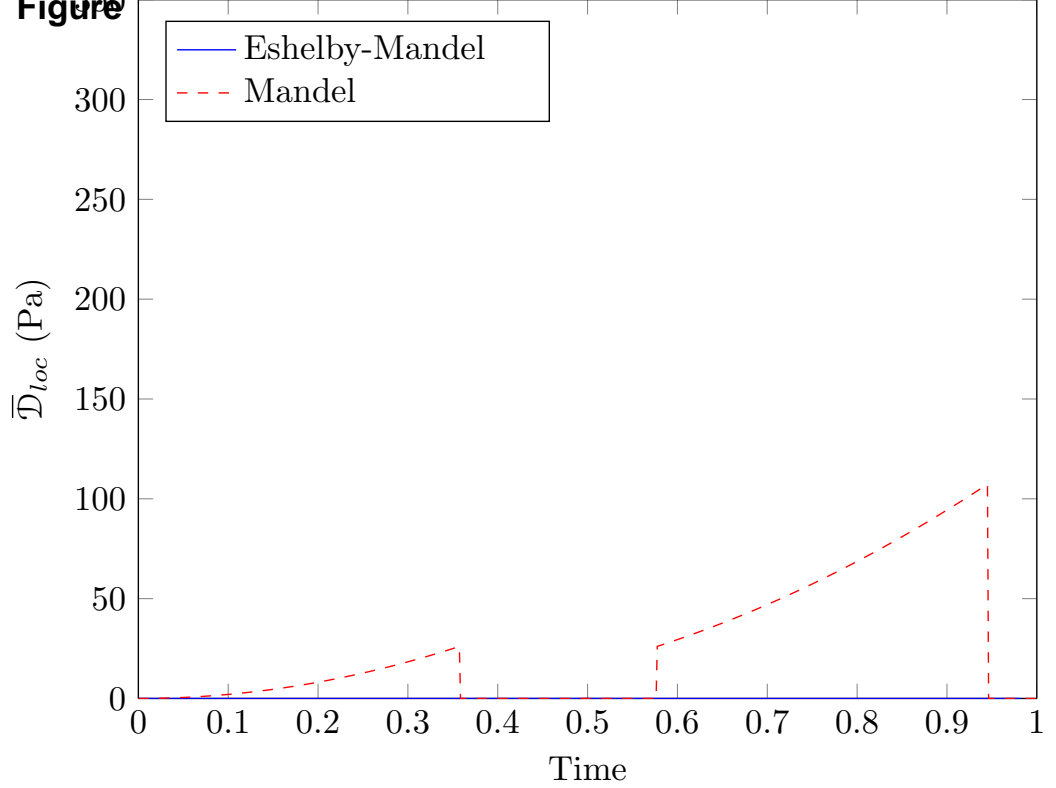


Figure $10^{8}$

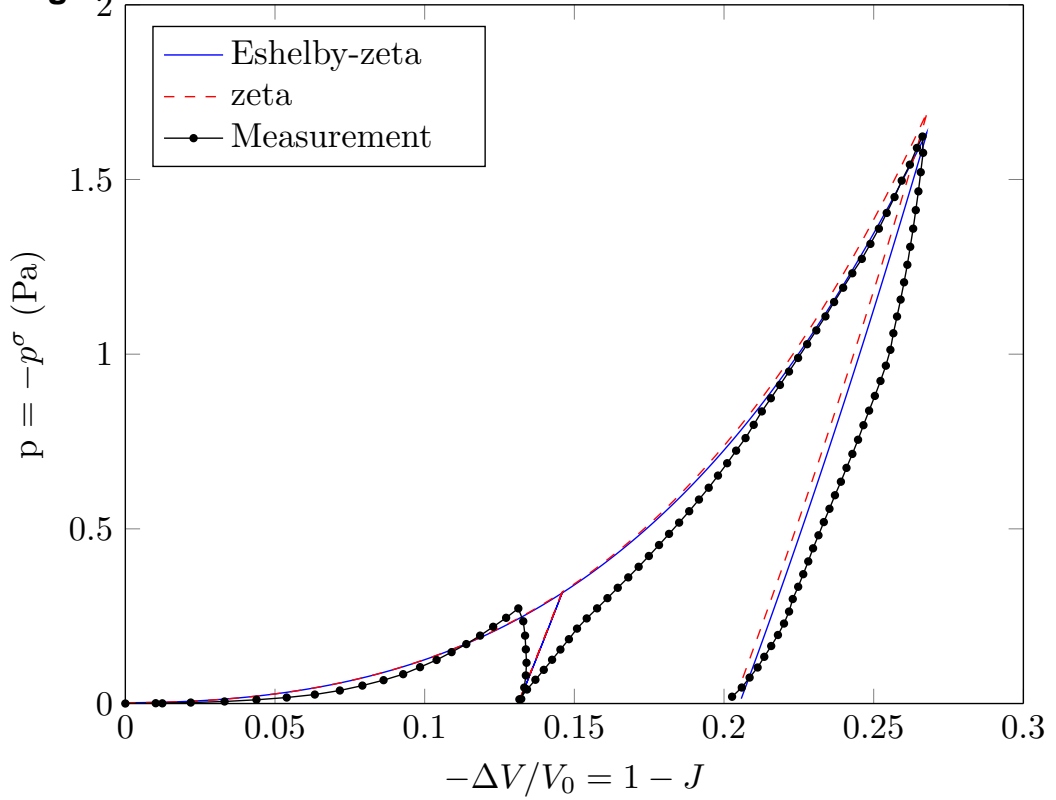


Figure $10^{8}$

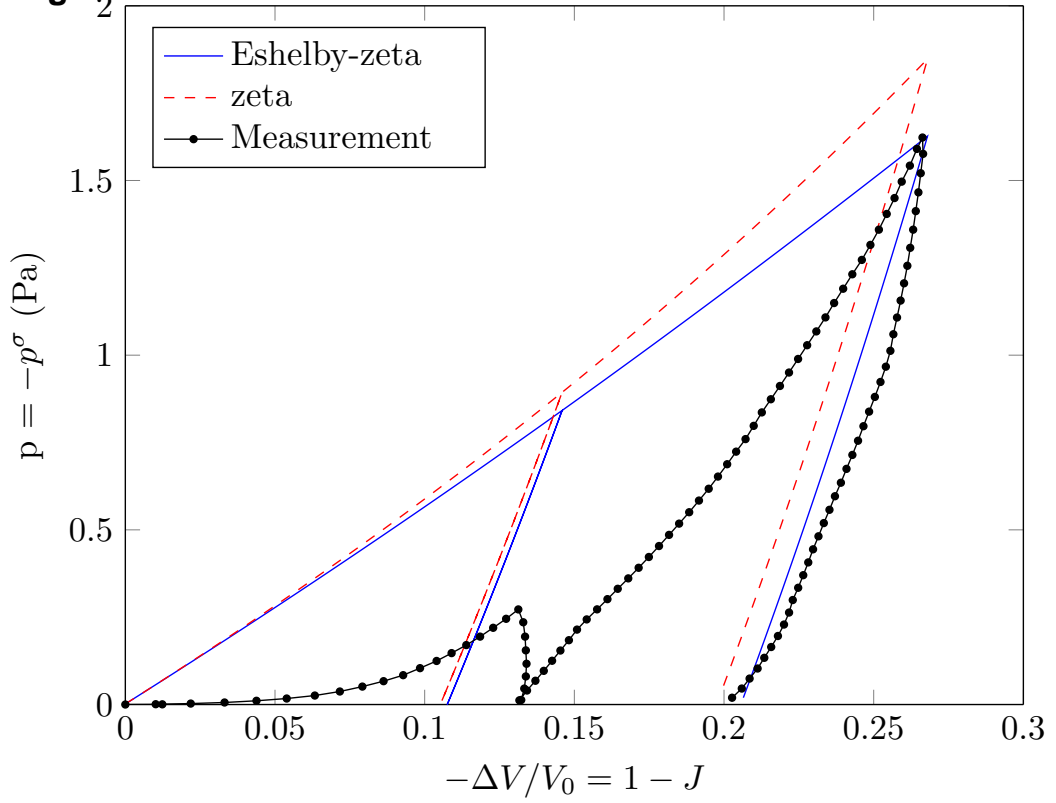




\section{Figurge}

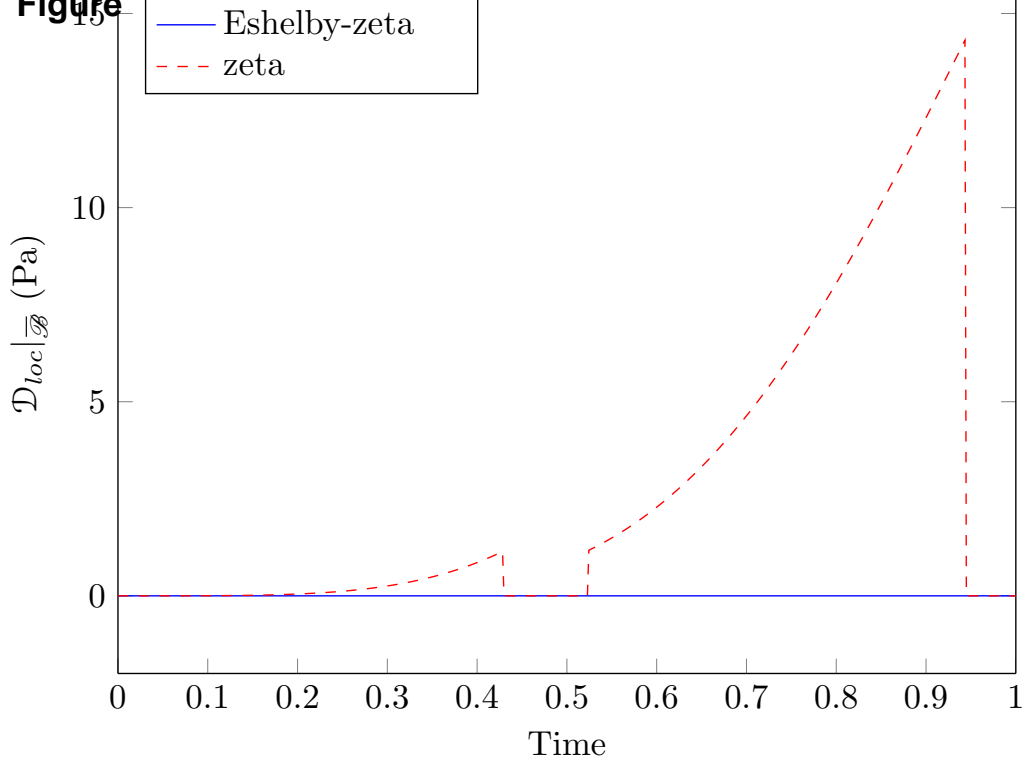




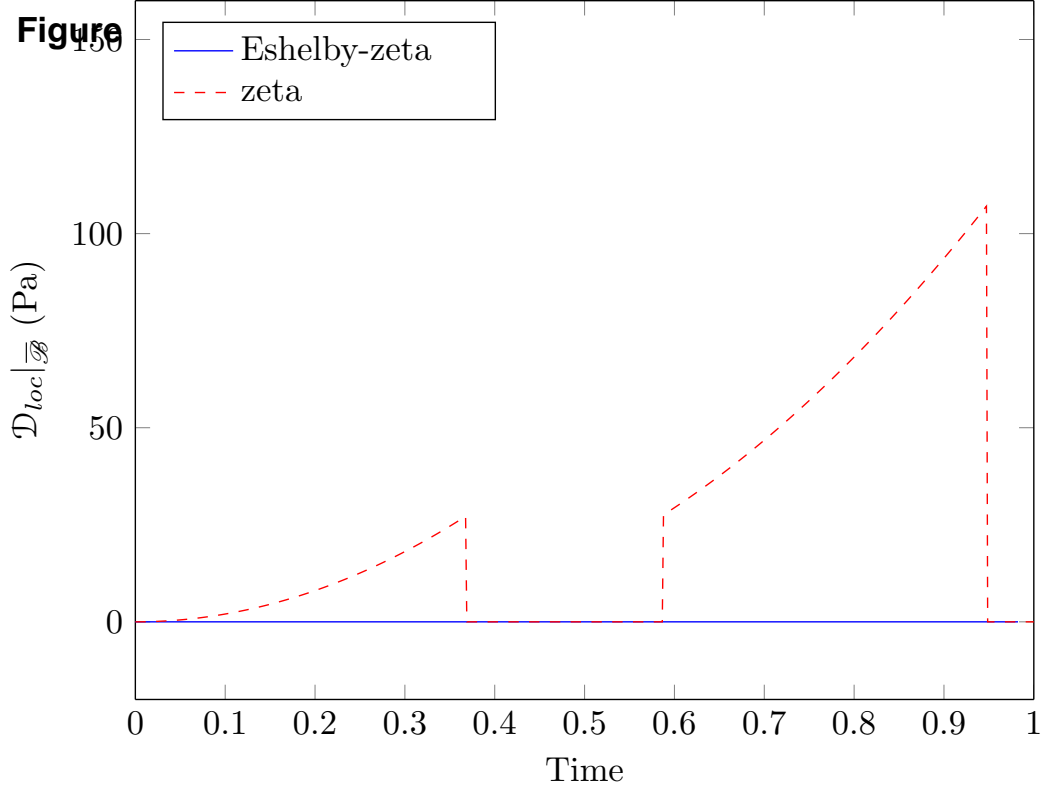


Figure $\cdot 10^{8}$

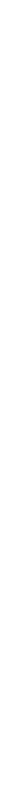


Figure $\cdot 10^{8}$

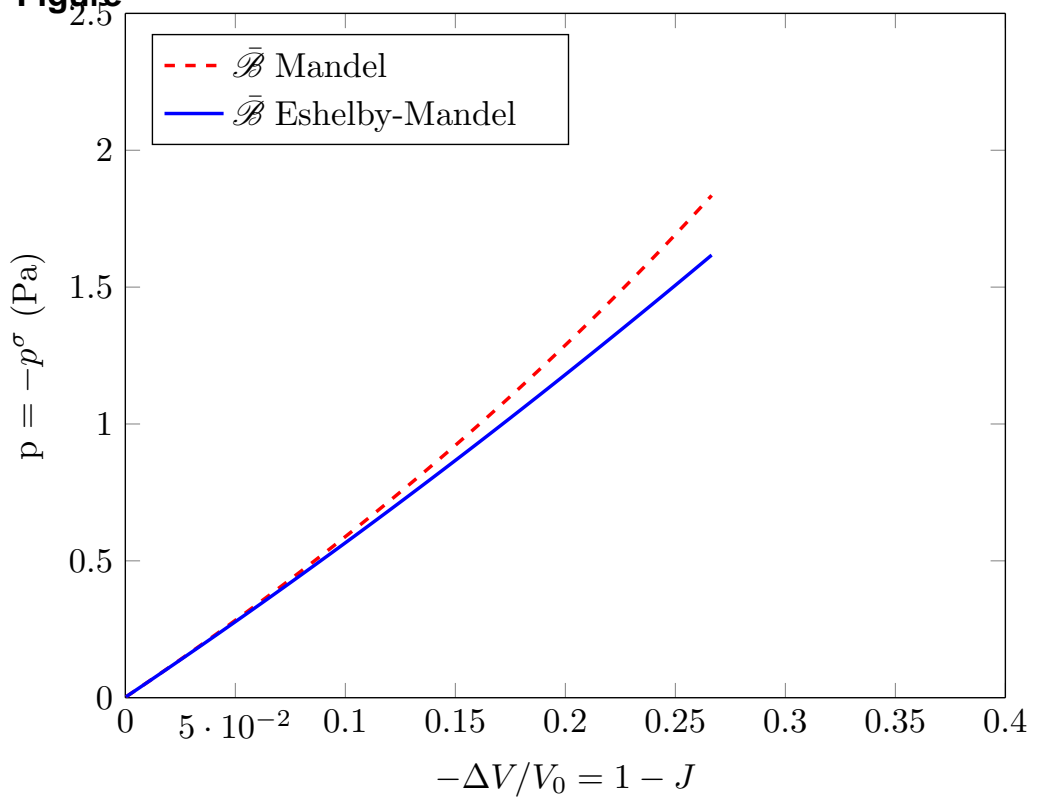


Figure $\cdot 10^{8}$

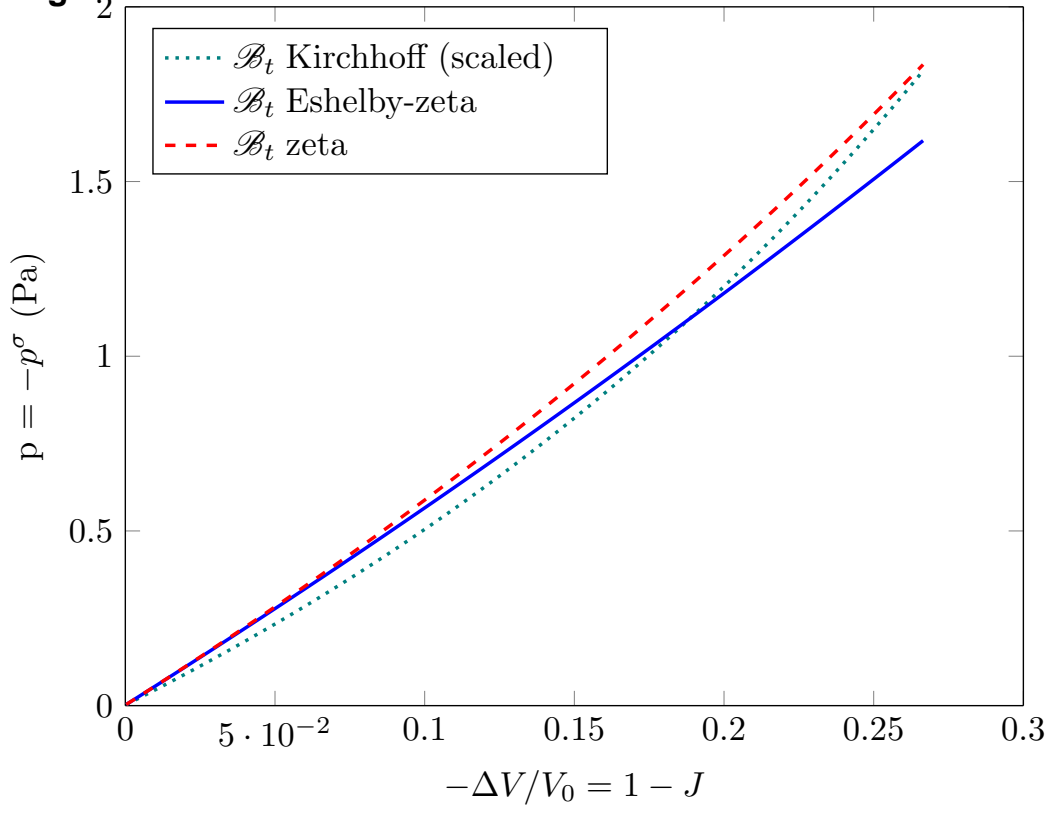




\section{Fighree}

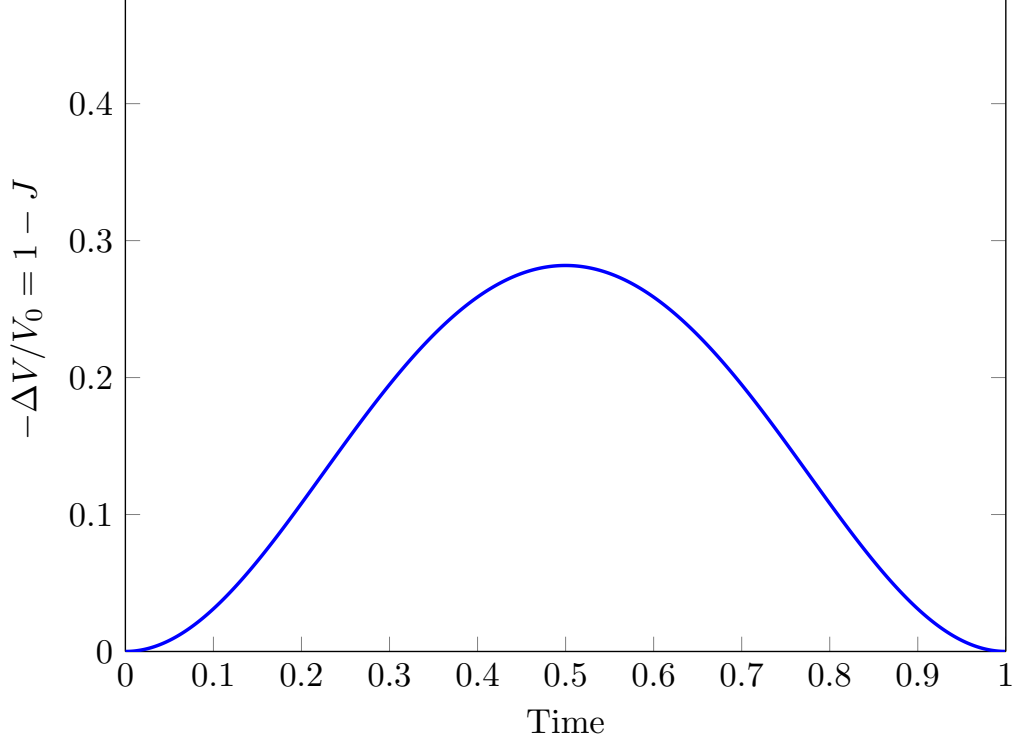


Figure $_{2} \cdot 10^{8}$

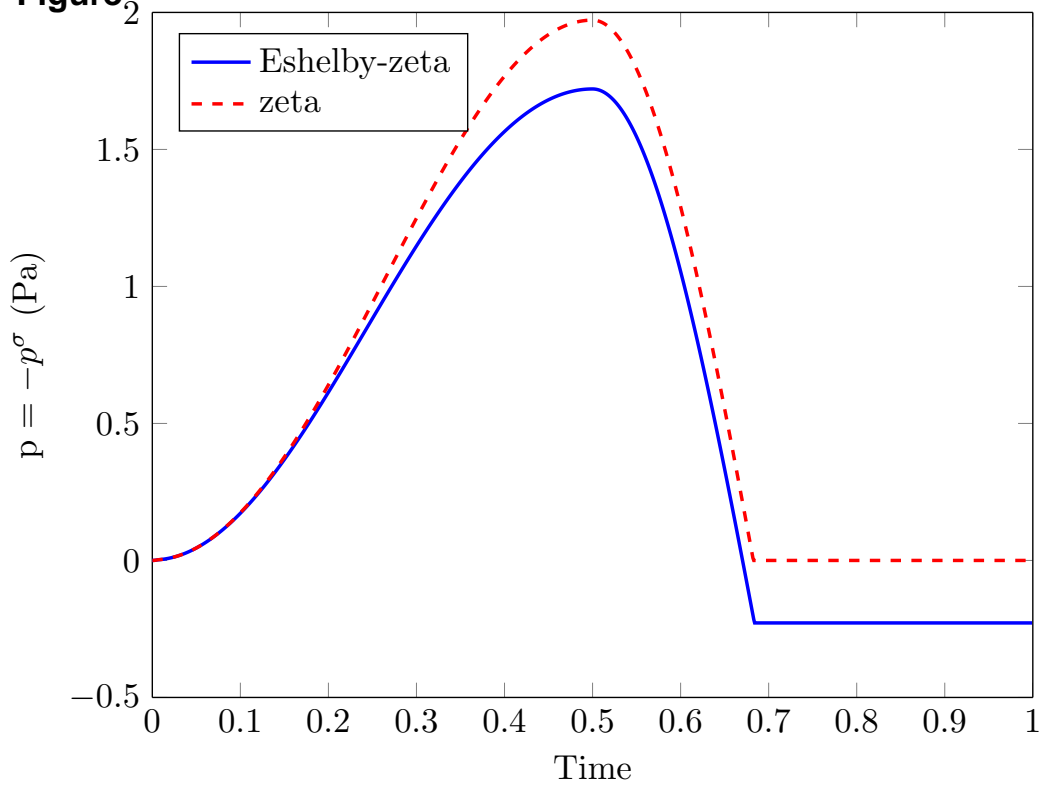


Figure ${ }_{0} \cdot 10^{8}$

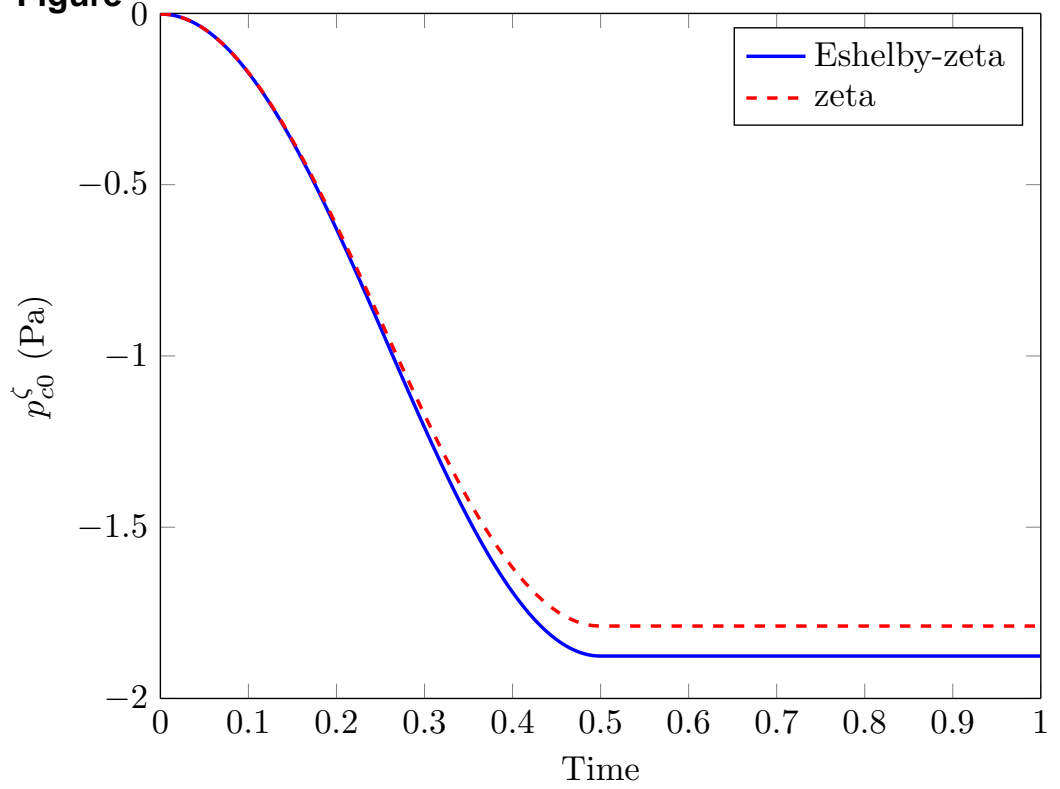




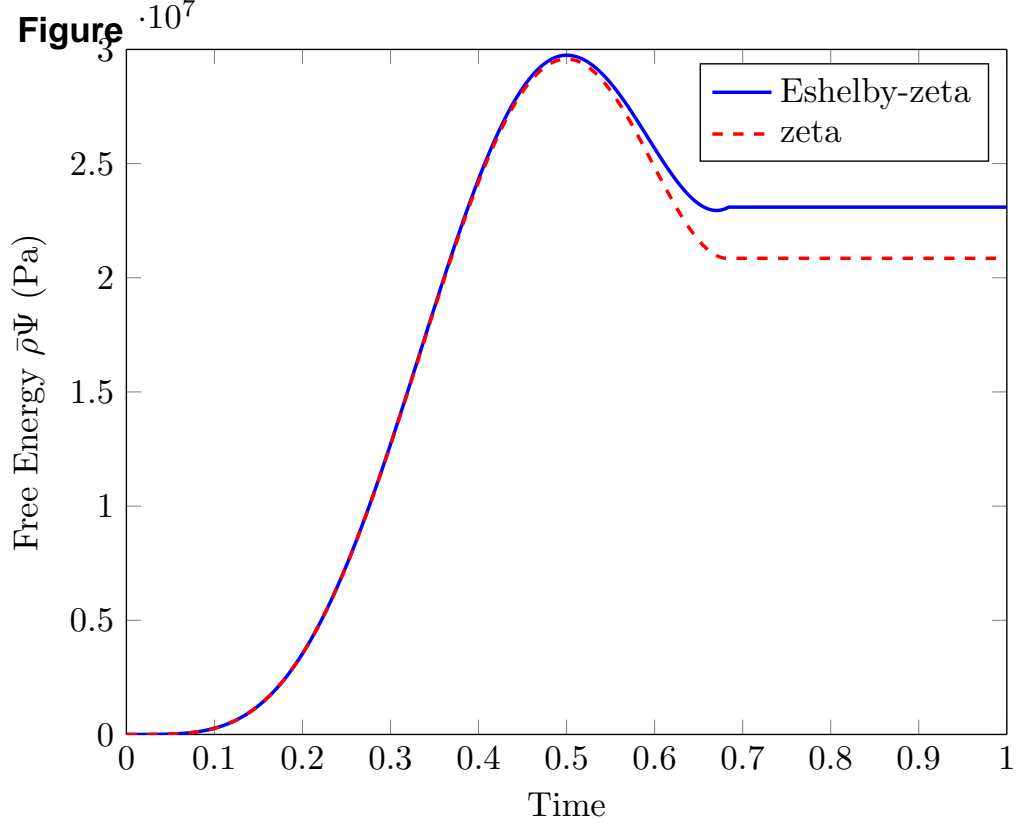




\section{Figure}

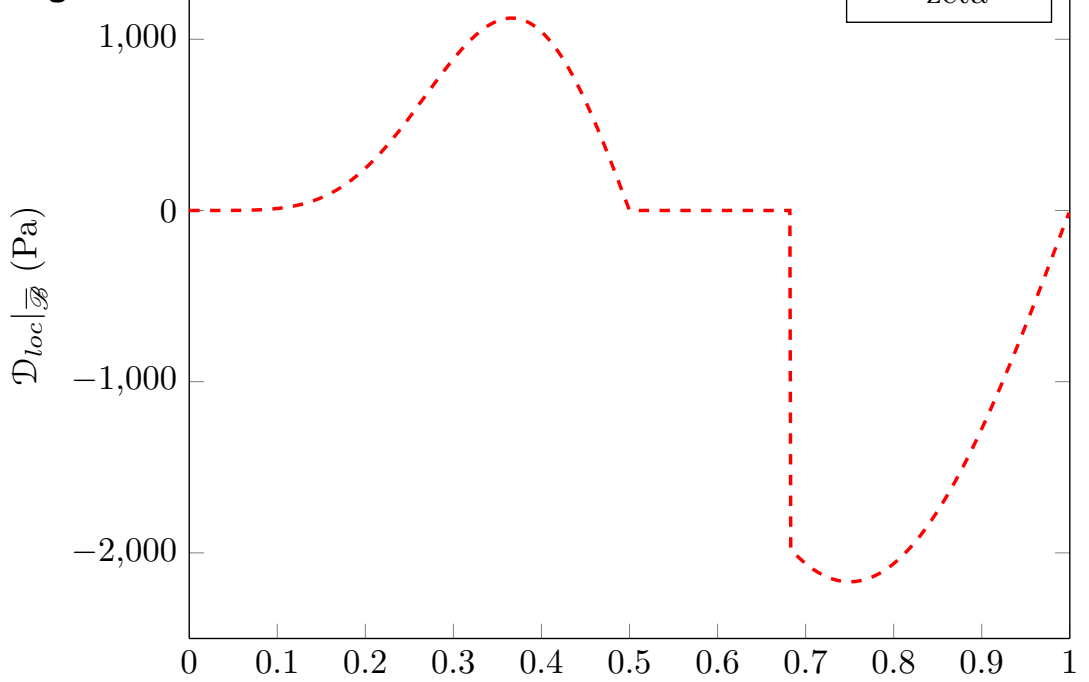

Time 